\author{
UNIVERSIDADE DE SÃO PAULO \\ FACULDADE DE ODONTOLOGIA DE BAURU
}

RHAELLEN CRISTINE SEVILHA SENIS

\title{
Avaliação do esforço auditivo com uso da amplificação por meio de paradigma de dupla tarefa e pupilometria
}



RHAELLEN CRISTINE SEVILHA SENIS

\section{Avaliação do esforço auditivo com uso da amplificação por meio de paradigma de dupla tarefa e pupilometria}

Dissertação apresentada à Faculdade de Odontologia de Bauru da Universidade de São Paulo para obtenção do título de Mestre em Ciências no Programa de Pós Graduação, na área de Fonoaudiologia.

Orientadora: Profa. Dra Wanderléia Quinhoneiro Blasca

Versão Corrigida 
Senis, Rhaellen Cristine Sevilha

Avaliação do esforço auditivo com uso da amplificação por meio de paradigma de duplatarefa e / Rhaellen Cristine Sevilha Senis. -- Bauru, 2020.

78p. : il. ; $31 \mathrm{~cm}$.

Dissertação-- Faculdade de Odontologia de Bauru, Universidade de São Paulo, ano de defesa.

Orientadora: Profa. Dra. Wanderléia QuinhoneiroBlasca

Nota: A versão original desta dissertação encontra-se disponível no Serviço de Biblioteca e Documentação da Faculdade de Odontologia de Bauru - FOB/USP.

Autorizo, exclusivamente para fins acadêmicos e científicos, a reprodução total ou parcial desta dissertação/tese, por processos fotocopiadores e outros meios eletrônicos.

Assinatura:

Data:

Comitê de Ética da FOB-USP

Protocolo:99717118.8.0000.5417

Data: 04/12/2018 
ERRATA 

FOLHA DE APROVAÇÃO 



\section{DEDICATÓRIA}

Dedico este trabalho primeiramente a Deus por ser essencial em minha vida, por iluminar meu caminho, pela força, coragem e por ser o maior mestre que alguém pode conhecer.

Aos meus pais Natalina Teixeira Sevilha e Marcos Antonio Senis pela educação, dedicação, apoio e incentivo nas horas difíceis, de desânimo e cansaço.

Às minhas irmãs Helen Cristine Teixeira Sevilha Rufatto, Carla Sevilha Senis e Camila Sevilha Senis por fazerem parte e compartilharem das minhas melhores lembranças, sendo sempre presentes em minha vida.

Aos meus avós Aparecida Maria Teixeira Sevilha e Waldemar Sevilha por todo amor e carinho.

Ao meu namorado Gustavo Mateus por todo amor, paciência e companheirismo nessa jornada. 



\section{AGRADECIMENTOS}

A Faculdade de Odontologia de Bauru pela estrutura física, pelos profissionais e pela oportunidade de uma excelente formação.

A Prof ${ }^{a}$ Dra $^{\text {a }}$ Wanderleia Quinhoneiro Blasca que dedicou seu tempo e compartilhou sua experiência para que minha formação fosse também um aprendizado de vida.

Ao Profo Dr Sérgio que se manteve acessível e contribuiu efetivamente durante todo o desenvolver da pesquisa. Muito obrigada pela paciência e ensinamentos!

Aos pacientes e seus familiares, que, contribuíram de forma direta para conclusão do estudo e por todo carinho recebido.

A Clínica de Fonoaudiologia, Departamento Fonoaudiologia e seus funcionários por todo respeito, suporte e apoio nessa etapa.

Aos colegas do curso por todos os momentos, auxilio e conhecimentos compartilhados.

Aos alunos do Laboratório de Informação, Visão e Ação (LIVIA) do Departamento de Educação Física da Faculdade de Ciências da Universidade Estadual Paulista (UNESP - Campus de Bauru) por contribuírem na coleta dos dados e pela disponibilidade.

A todos que direta ou indiretamente contribuíram para a concretização de mais uma etapa. 

"Entrega o teu caminho ao Senhor: confia nEle, e Ele o fará.

(Salmos 37:5) 



\section{RESUMO}

Introdução e justificativa: No processo da comunicação, estão envolvidos fatores perceptivos e cognitivos. Na prática clínica, um aspecto da compreensão da fala importante a se considerar é o esforço auditivo, que se refere à atenção e aos recursos cognitivos necessários para compreender a fala, principalmente considerando o avanço da idade. A aplicação de medidas objetivas, subjetivas e fisiológicas do esforço auditivo, que levem em conta a capacidade cognitiva do indivíduo e as situações de vida real, podem fornecer meios sensíveis de diferenciar os resultados com o uso de aparelhos de amplificação sonora na população idosa. No contexto do processo de adaptação do aparelho de amplificação sonora individual no idoso, é necessário estabelecer expectativas realistas quanto ao desempenho de inteligibilidade de fala e à demanda de esforço auditivo. Objetivo: Analisar o desempenho obtido na avaliação do esforço auditivo em indivíduos idosos com deficiência auditiva e usuários de aparelho de amplificação sonora individual. Material e métodos: Foram analisados 297 prontuários e selecionados 11 indivíduos idosos matriculados na clínica de fonoaudiologia da FOB/USP que se encaixavam nos critérios de inclusão do estudo, o quais foram submetidos aos procedimentos de avaliação do esforço auditivo com e sem o uso da amplificação. A avaliação subjetiva foi realizada por meio do questionário Speech, Spatial and Qualities of Hearing Scale (SSQ). Como forma de avaliação comportamental, foram aplicados o paradigma de dupla tarefa e a medida fisiológica pupilometria. Resultados: Não houve diferenças estatisticamente significativas na comparação dos dados obtidos no paradigma de dupla tarefa e na pupilometria nas situações de tarefa secundária e de dupla tarefa com e sem o uso de AASI. Conclusão: Há necessidade de continuidade e aprimoramento de estudos para que sejam desenvolvidos protocolos de avaliação do esforço auditivo no idoso. Porém, este estudo não mostrou a pupilometria como um procedimento eficaz para a mensuração do esforço auditivo.

Palavras-Chave: Idoso; Perda auditiva; Auxiliares de audição; Pupila. 



\section{ABSTRACT \\ Auditory effort assessment using amplification through double-task paradigm and pupillometry}

Introduction and justification: The communication process involves perceptual and cognitive factors. In the clinical practice, auditory effort is an essential aspect of speech comprehension, which refers to the attention and cognitive resources needed to understand speech, especially considering age. Applying objective and physiological measures of hearing effort, which takes into account the individual's cognitive ability and real-life situations, can provide sensitive means of differentiating outcomes with the elderly population. In the context of the process of hearing aid fitting in the elderly, there is a need to establish realistic expectations regarding speech intelligibility performance and hearing effort demand. Objective: To analyze the hearing effort evaluation performance of elderly individuals who have hearing impairment and wear hearing aids. Material and methods: A total of 297 medical records were analyzed, and 11 elderly individuals enrolled in the University of São Paulo's Bauru School of Dentistry (FOB USP) Speech-Language Pathology and Audiology clinic who met the study inclusion criteria were selected. After signing the informed consent form, they were submitted to auditory effort evaluation procedures with and without the use of amplification. Subjective assessment was performed by using the Speech, Spatial and Qualities of Hearing Scale (SSQ) questionnaire. As a means of behavioral assessment, the dual-task paradigm and the physiological pupillometry measurement were applied. Results: There were no statistically significant differences when comparing the data obtained in the dual task paradigm and pupillometry in the situations of secondary task and double task with and without the use of hearing aids. Conclusion: There is a need for continuity and improvement of studies to develop protocols for assessing auditory effort in the elderly. However, this study did not show pupillometry as an effective procedure for measuring auditory effort.

Keywords: Elderly; Hearing Loss; Hearing aids; Pupil 



\section{LISTA DE ILUSTRAÇÕES}

\section{FIGURAS}

Figura 1 - Tabela de Snellen .......................................................... 41

Figura 2 - Eyetracker ............................................................... 44

\section{GRÁFICOS}

Gráfico 1 - Comparação do HINT na TP e PDT sem e com o uso do AASI 50 



\section{LISTA DE TABELAS}

Tabela 1 - Características dos participantes ......................................... 49

Tabela 2 - Comparação da paleta na TS e PDTcom e sem AASI ........... 50

Tabela 3 - $\quad$ Análise das Questões do Questionário SSQ.......................... 51 



\section{LISTA DE ABREVIATURA E SIGLAS}

AASI Aparelho de Amplificação Sonora Individual

ABA Academia Brasileira de Audiologia

ASHA American Speech and Hearing Association

CD Compact Disk

CEP Comitê de Ética em Pesquisa

COSI Client Oriented Scale of Improvement

HHIE Hearing Handicap Inventory for Elderly

dB Decibels

EEG Eletroencefalograma

EIA Encontro Internacional de Audiologia

FDA Federal Drug Administration

FOB Faculdade de Odontologia de Bauru

HHIA Hearing Handicap Inventory for Adults

HHIE Hearing Handicap Inventory for Elderly

HINT Hearing in Noise Test

MS Ministério da Saúde

S segundos

NPS Nível de Pressão Sonora

OMS Organização Mundial de Saúde

PDT Paradgma de Dupla Tarefa

S/R Relação Sinal x Ruído

SSQ Speech, Spatialand Qualitie sof Hearing Scale

SUS Sistema Único de Saúde

TCLE Termo de Consentimento Livre e Esclarecido

TP Tarefa Primária

TS Tarefa Secundária

USP Universidade de São Paulo 



\section{SUMÁRIO}

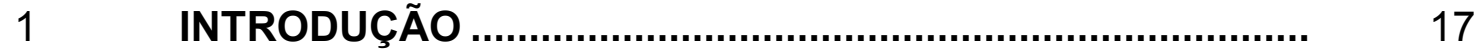

$2 \quad$ REVISÃO DE LITERATURA

2.1 PRESBIACUSIA: ENVELHECIMENTO E COGNIÇÃO .......... 23 ADAPTAÇÃO DE APARELHOS DE AMPLIFICAÇÃO SONORA

$2.2 \quad 25$ INDIVIDUAL NO IDOSO.

2.3 ESFORÇO AUDITIVO E PERCEPÇÃO DE FALA NO IDOSO 28

$3 \quad$ PROPOSIÇÃO …............................................................. 35

$4 \quad$ MÉTODOS ........................................................................ 39

$4.1 \quad$ ASPECTOS ÉTICOS ….................................................. 39

4.2 DELIMITAÇÃO DA CASUISTICA ....................................... 39

$4.3 \quad$ INSTRUMENTOS E PROCEDIMENTOS ............................. 42

4.3.1 MEDIDA COMPORTAMENTAL ........................................... 42

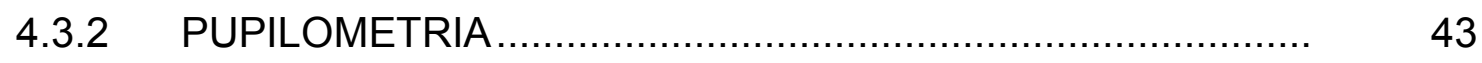

4.3.3 QUESTIONÁRIO .........................................................

$4.4 \quad$ ANALISE DOS RESULTADOS ………........................... 45

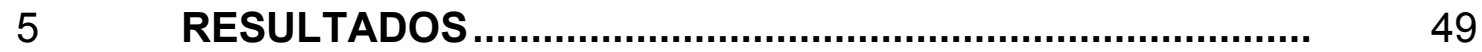

5.1 CARACTERIZAÇÃO DA AMOSTRA ………….................... 49

5.2 ESFORÇO AUDITIVO ........................................................ 49

5.2.1 MEDIDA COMPORTAMENTAL .......................................... 49

5.2 .2 PUPILOMETRIA ………….......................................... 51

5.2.3 QUESTIONÁRIO ...................................................... 51

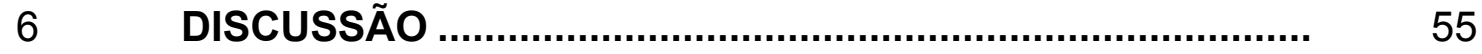

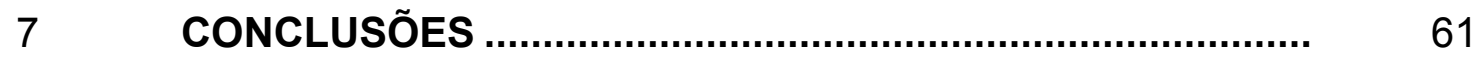

REFERÊNCIAS ............................................................ 65

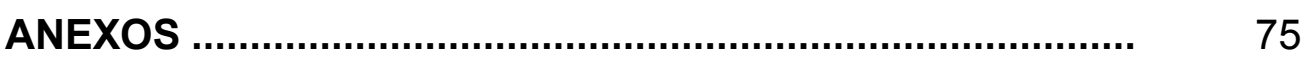



Introdução 



\section{INTRODUÇÃO}

O Brasil passa atualmente por um período de crescimento da população idosa. Segundo projeção realizada pelo IBGE para 2028, espera-se alcançar um total superior a 38 milhões de pessoas com mais de 60 anos de idade, ou seja, haverá um aumento maior do que o dobro da população idosa atual (IBGE, 2008).

O contexto do envelhecimento envolve o declínio das funções de todo o corpo. De forma bastante complexa, compreende aspectos físicos, psicológicos e sociais. Acontecendo de maneira diferente em cada indivíduo, a capacidade sensorial se apresenta frágil e deficiente.

A perda neurossensorial encontrada nos idosos, chamada de presbiacusia, é caracterizada como uma consequência frequente do processo de envelhecimento, sendo que a deficiência auditiva nesta população é uma das três condições crônicas mais prevalentes, perdendo apenas para a artrite e para a hipertensão (BESS et al., 2001).

A queixa mais referida pelo indivíduo com perda auditiva é que há dificuldade na compreensão do discurso, especialmente em ambientes ruidosos, os quais na vida diária representam a regra, e não a exceção (STEWART; WINGFIELD, 2009; PICHORA-FULLER; SOUZA, 2003). Essa condição envolve também lentificação do processamento central de estímulos auditivos e dificuldade na localização de fontes sonoras (KIM; CHUNG, 2013).

Além de gerar um grande impacto no processo de comunicação, a perda auditiva pode acarretar também prejuízos na interação do idoso na sociedade. Fatores sociais envolvidos, como comportamentos depressivos e de isolamento social, muitas vezes podem ser decorrentes da própria perda auditiva, que dificulta o processo de comunicação e, consequentemente, a vida em sociedade. Da mesma maneira, existem dificuldades sociais em relação ao estereótipo do deficiente auditivo em detrimento das dificuldades de comunicação (MARINI, HALPEN, AERTS, 2005; MURPHY, DANEMAN, SCHNEIDER, 2006; ZAZOVE et al., 2006).

Com o objetivo de evitar o isolamento social do idoso, uma das formas de amenizar as consequências da presbiacusia é utilizar o aparelho de amplificação sonora individual (AASI). Nesse contexto, o uso do AASI visa minimizar esses 
prejuízos decorrentes da perda auditiva e evitar sentimentos decorrentes das dificuldades geradas por essa deficiência. Assim, estudos referentes ao impacto da deficiência auditiva na vida do idoso e a percepção da queda da acuidade auditiva decorrente do envelhecimento são de grande importância para a qualidade de vida dessa população.

Com o avanço da idade, há igualmente um declínio das funções sensoriais, perceptivas e cognitivas do indivíduo (PICHORA-FULLER; SINGH, 2006). O uso do AASI está associado à melhora da cognição. Os aparelhos auditivos podem melhorar o desempenho auditivo, embora não seja provável que essa melhora aconteça devido à redução dos efeitos da perda auditiva nos aspectos sociais e emocionais, e sim por conta da melhora na audibilidade ou pela crença na própria capacidade de realizar tarefas ou de alcançar metas (DAWES et al., 2015).

O processo de reabilitação é complexo e deve ser realizado de forma a considerar as características e as dificuldades individuais, especificamente na população idosa. Dessa forma, o diagnóstico complementar com testes que proporcionem situações semelhantes às encontradas no cotidiano e que forneçam informações sobre esses aspectos, como o esforço auditivo, podem contribuir para a melhor forma de reabilitação.

Não há consenso na literatura sobre a melhor forma de mensurar o esforço auditivo, sendo todas consideradas válidas. Para tanto, são utilizadas medidas de autoavaliação, fisiológicas e/ou comportamentais (MCGARRIGLE et al., 2014, GAGNÉ et al., 2017).

Recentemente, uma das formas fisiológicas utilizadas é a pupilometria. A dilatação da pupila sofre influência da carga cognitiva empregada no esforço de escuta e tem sido utilizada para avaliação de reconhecimento de fala em ambientes auditivos difíceis (GOLDWATER, 1972; ZEKVELD; KRAMER; FESTEN, 2010)

O paradigma de dupla tarefa tem sido bastante utilizado e se mostrado eficaz para avaliar o esforço auditivo (PALS, SARAMPALIS, BAŞKENT, 2013). Esse paradigma consiste de duas tarefas: a primária é a percepção de fala, e a secundária é uma tarefa de memória de cores previamente apresentadas pelo sujeito que está sendo avaliado. Ambas são desempenhadas concomitantemente.

Diante do apresentado, observa-se que o diagnóstico audiológico deve ser realizado com o objetivo de auxiliar na seleção e na adaptação do AASI, além de definir estratégias de reabilitação auditiva. Porém, é necessário um estudo com 
procedimentos de avaliação complementar do esforço auditivo no idoso, objetivando a criação de estratégias de comunicação pautadas em expectativas reais no processo de reabilitação e proporcionando maior adesão ao tratamento, melhora na comunicação e, consequentemente, melhor qualidade de vida para os usuários de AASI.

Dessa forma, busca-se analisar a eficiência do uso do AASI na população idosa e descobrir se ocorre alteração do esforço auditivo. Esse esforço deve ser avaliado por meio do paradigma de dupla tarefa, aplicando-o em situações de escuta no ruído. Para esse monitoramento, a pupilometria deve ser o instrumento testado para comprovação da alteração da pupila frente à dificuldade de tarefa de escuta. 



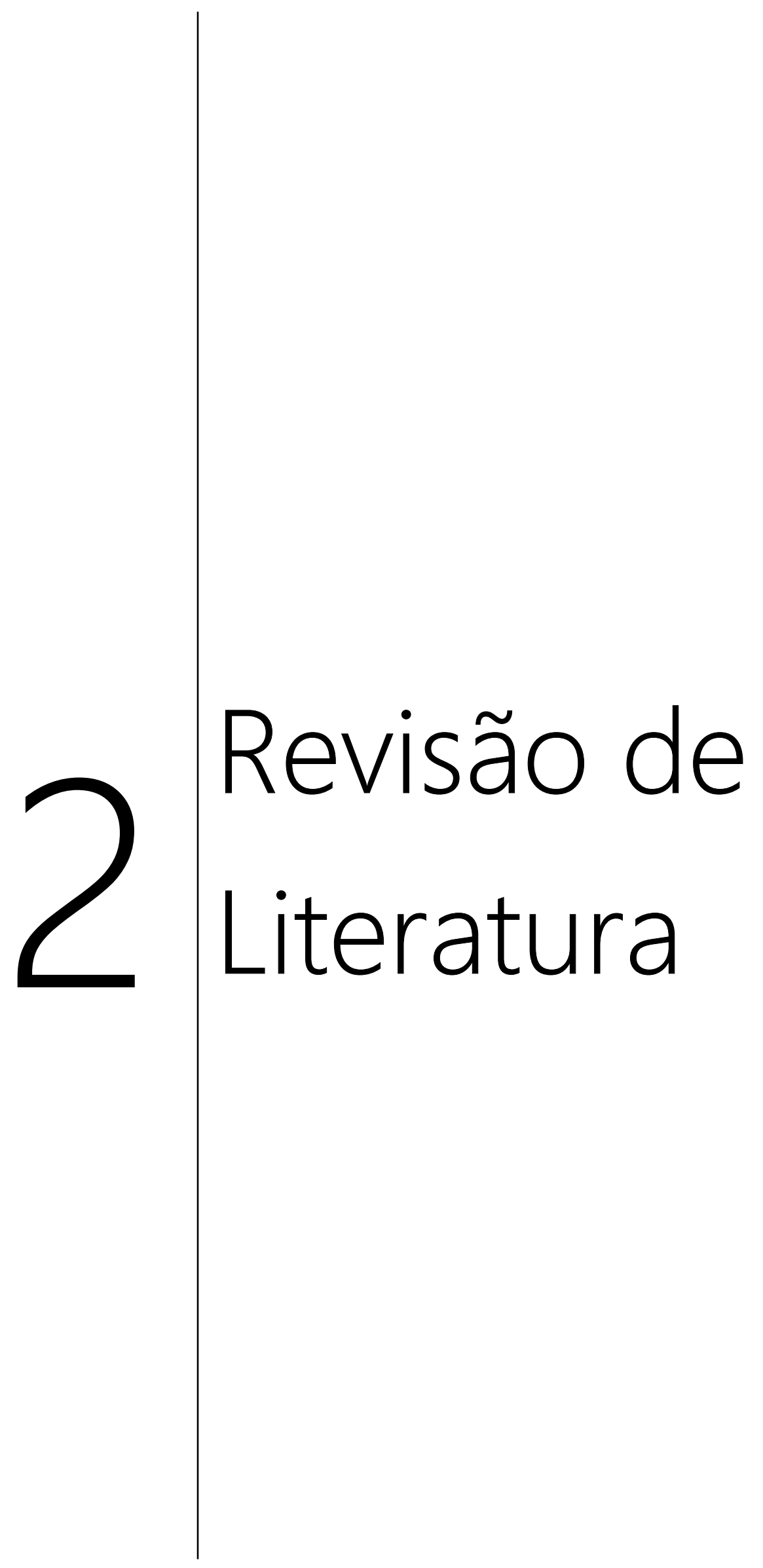





\section{REVISÃO DE LITERATURA}

\subsection{PRESBIACUSIA: ENVELHECIMENTO E COGNIÇÃO}

O envelhecimento pode ser definido como um processo dinâmico e progressivo, no qual há modificações morfológicas, funcionais, bioquímicas e psicológicas que determinam perda da capacidade de adaptação do indivíduo ao meio ambiente, ocasionando maior vulnerabilidade e maior incidência de processos patológicos (PAPALEO NETTO, 2011). Dentre as alterações decorrentes do envelhecimento, podemos citar a presbiacusia, que é um termo geral aplicado à perda auditiva relacionada ao envelhecimento (GATES; MILLS, 2005).

A presbiacusia é uma perda gradual e bilateral da audição associada ao envelhecimento, causada pela degeneração progressiva das estruturas cocleares e vias auditivas centrais (REIS; ESCADA, 2016). De fato, a sensibilidade para as frequências mais altas começa a diminuir na maioria dos indivíduos por volta dos 30 a 40 anos de idade, atingindo progressivamente as frequências mais baixas do espectro sonoro em fases mais tardias (MOORE et al., 2014).

Com o envelhecimento populacional, estima-se que em 2050 por volta de $59 \%$ dos casos da audiologia geral serão compostos de idosos (WORRALL; HICKSON, 2003), já que aproximadamente de dois terços das pessoas com 70 anos de idade ou mais têm perda auditiva (LIN et al., 2011).

A principal queixa dos idosos com perda auditiva está relacionada com a compreensão, principalmente quando há ruído competitivo com a fala. $A$ idade é um fator importante a ser considerado nessa habilidade, visto que, à medida que os adultos envelhecem, suas funções sensoriais, perceptivas e cognitivas diminuem (BALTES; LINDENBERGER; BALTES, 1994; PICHORA-FULLER; SINGH, 2006; SCIALFA, 2002).

Esse aspecto é observado também em idosos sem perda auditiva (CHABA, 1988). Isso sugere que, além de fatores perceptivos como a capacidade de ouvir, fatores cognitivos também são essenciais para a comunicação.

O declínio cognitivo pode estar relacionado à audição. Lin et al. (2011) pesquisaram 347 indivíduos com idade igual ou superior a 55 anos, que passaram por 
avaliação audiológica e neurocognitiva, sem demência ou défice cognitivo prévios. Os resultados mostraram que as funções cognitivas testadas diminuíam de forma linear aos níveis de perda auditiva, sendo as áreas mais afetadas a memória e a função executiva. Observou-se que a velocidade de processamento e a função verbal eram preservadas. Concluíram que a relação é significativa, tendo sido demonstrado que a diminuição da capacidade cognitiva associada a uma perda auditiva de $25 \mathrm{~dB}$ é equivalente a uma diferença de 6,8 anos nos resultados dos testes de função cognitiva.

Em outro estudo, os autores Lin e Metter (2011) analisaram 639 participantes com idades entre 36 e 90 anos e concluíram que o risco de demência aumenta linearmente com o nível de perda auditiva, sendo mais evidente a partir dos limiares acima de $25 \mathrm{~dB}$, que começa a interferir com a compreensão verbal, segundo a Organização Mundial da Saúde (OMS), e aumenta linearmente de acordo com o grau da perda auditiva. Quando foram comparados os indivíduos com perda auditiva com aqueles com função auditiva preservada, verificou-se que aqueles com perda auditiva leve, moderada e severa tinham duas, três e cinco vezes maior risco de desenvolver demência, respetivamente.

Gallacher et al. (2012) avaliaram durante 17 anos as capacidades auditiva e cognitiva de 1057 indivíduos relacionadas à presença de demência. O estudo mostrou que houve relação forte entre perda auditiva, declínio cognitivo e demência.

Os prejuízos da perda auditiva no idoso, alterações na cognição, dificuldades na compreensão de fala e, consequentemente, na comunicação fazem com que o paciente conviva menos em sociedade. A socialização é um aspecto muito relevante na terceira idade, quando é muito comum o idoso sentir-se isolado, pois muitas vezes as pessoas ao seu redor estão voltadas para interesses diferentes. A dificuldade de inserção grupal leva o idoso a se fechar em seus pares ou a se isolar socialmente, evitando os conflitos que possam surgir desta diversidade de interesses e de hábitos entre ele e as gerações mais novas (VIEIRA, 2004).

$O$ idoso adquire, também, o sentimento de constrangimento perante sua dificuldade de ouvir, o que pode propiciar o surgimento de um quadro depressivo. Além dos problemas de saúde física, idosos têm aumento da prevalência de sintomas depressivos (SCHOEVERS, 2000; MILLS, 2001), estando mais expostos a abuso e negligência por parte de seus familiares e prestadores de cuidados (GIURANI; HASAN, 2000). 
Por isso, é necessário que haja um processo de identificação e de tratamento das alterações senis. No âmbito da perda auditiva, o aparelho auditivo pode ser uma alternativa eficiente.

A adaptação do AASI tende a diminuir as dificuldades decorrentes da perda auditiva, visto que a amplificação permite que o idoso possa ter acesso à sensação dos sons. Nessa idade, a busca pela melhoria da qualidade de vida mostra que a população idosa procura se prevenir dos efeitos deletérios do processo de envelhecimento humano. As alterações de aspectos físicos, psicológicos e sociais decorrentes do processo de envelhecimento afirmam a influência que possuem na capacidade funcional na terceira idade. Os aspectos biopsicossociais têm grande importância em todo esse processo na terceira idade (LIMA; DELGADO, 2010).

\subsection{ADAPTAÇÃO DE APARELHOS DE AMPLIFICAÇÃO SONORA INDIVIDUAL NO IDOSO}

Um dos principais fatores que contribuem para a qualidade de vida do idoso é a manutenção da capacidade de comunicação. Por meio da comunicação, o indivíduo mantém suas relações sociais, sendo a linguagem falada uma das mais utilizadas no mundo para as trocas comunicativas. Para que ela ocorra adequadamente, é necessária, dentre outros aspectos, a preservação da audição, pois sua privação representa uma das causas de isolamento social para o idoso (CARMO et al., 2008; MONDELLI; SOUZA, 2012).

Algumas alterações além da diminuição da audição devem ser consideradas, dentre elas a incapacidade auditiva e a desvantagem auditiva (MARQUES; KOZLOWSKI; MARQUES, 2004). A incapacidade auditiva está relacionada à falta de habilidade de percepção de fala em ambientes ruidosos ou na televisão, por exemplo. Já a desvantagem auditiva refere-se aos aspectos não auditivos, os quais impedem o indivíduo de desempenhar adequadamente seu papel na sociedade (RUSSO; ALMEIDA, 1995).

A presbiacusia pode contribuir para o desenvolvimento de alguns distúrbios psiquiátricos, favorecendo o isolamento dos portadores da deficiência devido à dificuldade de comunicação com o meio social em que vivem. Os familiares do deficiente, muitas vezes, não têm tolerância para lidar com a falta de audição e, 
normalmente, não mantêm diálogos normais com o idoso, passando somente a informar os assuntos essenciais (MONDELLI; SOUZA, 2012).

Após o diagnóstico da perda auditiva nessa população, uma alternativa de tratamento possível, em muitas situações, é o uso de AASI (MONDELLI; SOUZA, 2012; MARQUES; KOSLOWSKY; MARQUES, 2004). O AASI é um dispositivo eletrônico que tem como função amplificar o som de modo a permitir que se utilize o resíduo auditivo de maneira efetiva, proporcionando auxílio para o entendimento da fala e para a percepção dos sons ambientais nos portadores de deficiência auditiva (BLASCA, 2006).

Conforme a diretriz estabelecida pela Federal Drug Administration (FDA), bem como a motivação para a reabilitação auditiva (ASHA, 1998, VALENTE et al., 2006), a avaliação audiológica adequada pode proporcionar identificação do tipo e do grau da perda auditiva; determinação ou não da intervenção medicamentosa e/ou cirúrgica; devolutiva dos resultados audiométricos; as opções adequadas para o indivíduo, família ou cuidador por meio de aconselhamento; determinação de se o indivíduo é candidato à amplificação e orientação do plano de tratamento para esse indivíduo; e determinação da necessidade de afastamento médico.

A Academia Americana de Audiologia (AMERICAN ACADEMY OF AUDIOLOGY, 2008) sugere o uso do protocolo de seleção e de adaptação de AASI em adultos desenvolvido pela American Speech and Hearing Association (ASHA, 2008) para garantir a qualidade e segurança dos usuários. Sua realização ocorre conforme descrita a seguir:

1. Avaliação do indivíduo

- Avaliação auditiva: histórico do caso, inspeção visual da orelha externa, avaliação audiológica e pesquisa dos níveis de desconforto;

- Avaliação das necessidades de comunicação e de desempenho: questionários de autoavaliação Hearing Handicap Inventory for the Elderly (HHIE), Hearing Handicap Inventory for Adults (HHIA), Abbreviated Profile of Hearing Aid Benefit (APHAB) e Client Oriented Scale of Improvement (COSI);

- Avaliação das necessidades não auditivas: declínio cognitivo, características de personalidade, déficits sensoriais associados, experiência anterior com AASI, saúde geral e outras condições auiológicas como zumbido. 
2. Aspectos técnicos do tratamento

- Seleção do AASI:

- Característica eletroacústica: ganho, saída, entrada-saída;

- Outras: adaptação uni ou bilateral, processamento do sinal, controle de volume, bobina de indução;

- Controle de qualidade: inspeção visual, auditiva e avaliação eletroacústica (medidas em acoplador);

- Verificação:

- Adaptação física: estética, conforto, facilidade de inserção/remoção, ausência de realimentação acústica, segurança, localização do microfone, manutenção;

- Desempenho: audibilidade para sons fracos, conforto para sons médios e tolerância para sons fortes.

3. Orientação e aconselhamento:

- Orientação:

- Componentes, características e funcionamento do AASI; cuidado e mantenção; treinamento e manipulação, remoção e inserção do AASI; vida útil e troca de pilha; manipulação dos controles externos; cuidados gerais, limpeza, uso do telefone e rotina de uso de AASI;

- Aconselhamento:

- Anatomia e fisiologia da audição; compreensão do audiograma, problemas relacionados à compreensão de fala em ambientes ruidosos, comportamentos de escuta e estratégias de comunicação, controle do ambiente, expectativas e manejo do estresse, noções de leitura orofacial e uso de tecnologia assistida;

- Segmento:

- Aconselhamento pós-adaptação.

4. Avaliação dos resultados:

- Benefício objetivo: reconhecimento de fala no silêncio e ruído;

- Benefício subjetivo: limitações em atividades e restrição de participação;

- Satisfação e uso. 
No processo de seleção e de adaptação do aparelho de amplificação sonora individual, o sucesso está diretamente relacionado ao trabalho de orientação e de aconselhamento, mas o profissional precisa conhecer as reais dificuldades dos pacientes em relação ao uso adequado da amplificação (CAMPOS; OLIVEIRA; BLASCA, 2010).

Diante do envelhecimento populacional, o governo brasileiro tem desenvolvido políticas públicas de saúde. Em 2004, o Ministério da Saúde publicou a Portaria MS n 2.073, que instituiu a Política Nacional de Atenção à Saúde Auditiva no Brasil, e as portarias SAS/MS n 587 e 589, que determinaram as diretrizes para credenciamento de serviço de atenção básica, média e de alta complexidade para o atendimento da deficiência auditiva.

Os serviços credenciados seguem o Instrutivo de Saúde Auditiva Ref. Portaria GM 79 de 24 de abril de 2012 e a Portaria GM 835 de 25 de abril de 2012, que propõe diretrizes para tratamento e reabilitação/habilitação de pessoas com deficiência auditiva com recomendações quanto aos critérios de indicação de AASI de acordo com nas necessidades e classificação dos AASI com suas características eletroacústicas.

As políticas públicas em saúde auditiva no Brasil são um tema abordado nos fóruns anuais nos Encontros Internacionais de Audiologia (EIA) organizados pela Academia Brasileira de Audiologia (ABA). Isso mostra a importância e a preocupação em estabelecer condutas e serviços que atendam a necessidade da população.

\subsection{ESFORÇO AUDITIVO E PERCEPÇÃO DE FALA NO IDOSO}

O esforço auditivo refere-se à atenção e aos recursos cognitivos necessários para compreender a fala (BOURLAND-HICKS; THARPE, 2002) e à quantidade de recursos de processamento alocados a uma tarefa auditiva específica quando as demandas são altas e o indivíduo se esforça para alcançar um alto nível de desempenho. Sob condições de audição ideais, ouvir a fala relativamente não demanda esforço (RONNBERG et al., 2013). No entanto, o processamento da fala pode necessitar mais esforço quando a qualidade do sinal é degradada, quando a estrutura da linguagem utilizada é complexa ou quando o conteúdo da mensagem é menos familiar (GAGNÉ; BESSER; LEMKE, 2016). 
Os efeitos do envelhecimento sobre o esforço auditivo e a deficiência auditiva podem aumentar as dificuldades de audição, particularmente no ruído (WORRALL; HICKSON, 2003; LARSBY; HALLGREN; LYXELL, 2005; TUN; BENICHOV; WINGFIELD, 2008).

Humes e Coughlin (2009) avaliaram o esforço auditivo de adultos e idosos em situação de percepção de fala no ruído em conjunto fechado. Os participantes foram divididos em dois grupos: um de idosos com idade de 65 a 86 anos, com perda auditiva sensorioneural de característica descendente, e outro de adultos com idade entre 18 a 28 anos cuja audição era normal. Ambos os grupos foram avaliados com os mesmos estímulos. Os pesquisadores concluíram que os idosos tiveram pior desempenho do que os adultos jovens.

Tun, McCoy e Wingfield (2009) estudaram 48 indivíduos divididos em quatro grupos iguais separados por idade (adultos jovens e idosos) e acuidade auditiva (ouvintes normais e com perda auditiva de grau leve a moderado), com o objetivo de analisar o efeito do esforço auditivo sobre a memorização das listas de palavras por adultos mais jovens e mais velhos por meio de paradigma de dupla tarefa. Os achados sustentam a hipótese de que o esforço extra no nível sensorial-perceptivo que acompanha a perda auditiva tem consequências negativas para a memorização, um efeito que pode ser agravado com o envelhecimento.

É possível encontrar na literatura estudos que utilizam maneiras diversas para avaliação do esforço auditivo, sendo formas de autoavaliação, métodos comportamentais e fisiológicos os mais citados. Atualmente não há consenso quanto à melhor forma de avaliação, apesar de algumas formas se mostrarem eficazes (GAGNÉ et al., 2017).

O questionário Speech, Spatial and Qualities of Hearing Scale (SSQ) (GATEHOUSE; NOBLE, 2004) é uma maneira de autoavaliação que, apesar de não ter o objetivo de medir o esforço auditivo, inclui questões importantes que abrangem as situações do mesmo no cotidiano. Além disso, serve como forma complementar de avaliação, pois visa captar a perspectiva do indivíduo sobre o próprio quadro clínico, mostrando assim a percepção da sua condição, que não é, necessariamente, consistente com os dados coletados em outras avaliações (SANTANA, 2017).

O paradigma de dupla tarefa, uma abordagem comportamental que fornece uma medida quantitativa durante uma condição de escuta específica (BOURLANDHICKS; THARPE, 2002), tem sido bastante estudado e classificado como eficaz na 
medida do esforço auditivo. Em um paradigma de dupla tarefa, o indivíduo é solicitado a realizar duas tarefas, uma primária e uma secundária, separadamente e simultaneamente. A tarefa primária envolve uma atividade de audição, tal como reconhecimento de palavras no silêncio ou no ruído, e a tarefa secundária pode envolver memória, tempo de reação a um estímulo visual ou reconhecimento de padrão tátil (BOURLAND-HICKS; THARPE, 2002; FRASER et al., 2010). O esforço auditivo é calculado como a diferença de desempenho na tarefa secundária e a condição de dupla tarefa, ou seja, duas tarefas apresentadas simultaneamente (GAGNÉ; BESSER; LEMKE, 2016). A dificuldade da tarefa primária é sistematicamente variada, e a mudança no desempenho da tarefa secundária em relação a diferentes níveis de dificuldades de tarefa primária reflete em uma mudança nos recursos cognitivos e/ou auditivos para o processamento de fala (WU et al., 2014).

Heinrich, Henshaw e Ferguson (2016) utilizaram o paradigma de dupla tarefa como instrumento para investigar a relação entre a autoavaliação e a medida comportamental de percepção de fala. A tarefa primária contava com teste de fala e a tarefa secundaria com teste de memória. Foi aplicado em um grupo de 30 indivíduos com idade entre 50 e 74 anos, perda auditiva bilateral do tipo sensorioneural, de grau leve a moderado, usuários de AASI há pelo menos 3 meses. Os estímulos de fala foram apresentados em uma intensidade de $65 \mathrm{~dB}$, no silêncio ou com ruído de fundo, em duas relações sinal/ruído ( $\mathrm{S} / \mathrm{R})$ : $0 \mathrm{~dB}$ e $-4 \mathrm{~dB}$. A associação entre a percepção da fala e a cognição variou de acordo com os testes utilizados. Os resultados demonstraram que a tarefa de percepção de fala associada a uma tarefa de memória aumenta o esforço auditivo.

Gagné, Besser e Lemke (2017) realizaram um estudo de revisão de literatura sobre o esforço em situação de reconhecimento de fala em jovens e idosos no qual o paradigma de dupla tarefa foi utilizado como forma de mensuração. O estudo encontrou 29 trabalhos publicados no período entre 1982 e 2016. Os resultados concluíram que há grande variabilidade nas tarefas que compõem o paradigma experimental de dupla tarefa, não sendo possível determinar qual a melhor escolha para medir o esforço auditivo. No entanto, há o consenso entre os trabalhos revisados de que o paradigma de dupla tarefa é uma forma bem-sucedida de avaliar o esforço sob diferentes condições tanto em jovens quanto em idosos.

Guijo et al. (2018) realizaram um estudo de revisão de literatura a fim de descrever a grande variedade de abordagens metodológicas aplicadas, 
especialmente a variabilidade de tarefas secundárias que utilizam a capacidade de memória operacional para mensurar o esforço auditivo. Analisaram trabalhos publicados em bases de dados entre 2007 e 2017 e concluíram que os achados da revisão permitiram inferir que os diferentes métodos comportamentais empregados foram sensíveis a uma série de condições experimentais, como idade dos participantes, grau da perda auditiva, tipo de ruído empregado, tipo de estímulo de fala utilizado, capacidade de memória e algoritmos do AASI.

Cruz et al. (2019) desenvolveram a PALETA, uma tarefa de memorização de cores utilizada como instrumento de tarefa secundária para avaliar o esforço auditivo por meio do paradigma de dupla tarefa. Participaram do estudo 31 adolescentes com idade entre 12 e 18 anos divididos em dois grupos: o primeiro grupo com audição normal e o segundo com perda auditiva. Além disso, eram usuários de AASI e sistema de frequência modulada (sistema FM). As conclusões também sugerem que a PALETA é proposta como um procedimento de fácil aplicação como tarefa secundária, e os resultados da pesquisa revelaram que o uso do sistema FM reduz o esforço auditivo.

Uma forma fisiológica encontrada em estudos é a pupilometria, que mostrou refletir o esforço auditivo ao examinar como as mudanças no tamanho da pupila estão relacionadas às mudanças na atividade mental. $O$ tamanho da pupila, que não está sob controle cognitivo direto, pode ser monitorado discretamente com um eye tracker (metodologia que permite ao pesquisador acompanhar o olhar do usuário sobre uma interface ou objeto) ou por um pupilômetro ao longo de um experimento (KUCHINSKY; ECKERT; DUBNO, 2011).

Geralmente, quando uma tarefa requer mais carga de processamento no mesmo intervalo de tempo, a dilatação média da pupila é maior quando a tarefa está sendo realizada (GRANHOLM; VERNEY, 2004).

Kuchinsky et al. (2013) examinaram 21 idosos com idade entre 61 e 88 anos com perda auditiva sensorioneural de grau moderado e sem experiência prévia de uso de AASI. Os autores tinham o objetivo de verificar as mudanças na resposta da pupila com testes auditivos e lexicais. Os participantes identificaram palavras com dois níveis de relação sinal/ruído. As análises da curva de crescimento revelaram que a resposta da pupila foi afetada pelo aumento do ruído, que tornava a tarefa de percepção de fala mais difícil. A pupilometria detectou esses efeitos para ensaios corretos e ao longo do tempo de reação, sugerindo, com isso, que essa avaliação 
fornece evidências adicionais de dificuldade da tarefa quando comparada às medidas comportamentais.

Wagner, Toffanin e Baskent (2016) compararam os dados da pupilometria no processo automático de competição lexical entre discurso natural e degradado e combinaram fixações de olhos, que capturam o curso de desambiguação lexical, em 73 adultos com audição normal e com idade entre 20 e 31 anos. Concluíram que a degradação do sinal ou do canal receptor pode ocasionar um aumento do esforço mental. Além disso, o processamento incompleto e com esforço nos primeiros estágios pré-lexicais tem suas consequências no processamento lexical, pois agrega incerteza à informação.

McMahon et al. (2016) compararam o poder alfa e dilatação da pupila em uma tarefa de percepção de sentenças em 15 níveis aleatórios de $S / R$ (de $-7 d B$ a $+7 \mathrm{~dB}$ ) em duas condições: altamente inteligivel (16 canais) e moderadamente inteligível (6 canais). Participaram da pesquisa 16 adultos com audição normal e idade entre 19 e 28 anos. O eletroencefalograma (EEG) foi realizado em 16 participantes, e a pupilometria em 10 participantes. Os resultados mostram que esses métodos podem não ser eficientes e bem compreendidos como forma de avaliação dos processos cognitivos envolvidos no esforço auditivo em cenário clínico.

Outro estudo encontrado na literatura que usou a pupilometria como instrumento de avaliação investigou o curso do tempo de competição lexical e integração semântica ao processar fala degradada em 28 adultos falantes de holandês, com audição normal e com idade entre 20 e 30 anos. Os achados mostram que a integração semântica inicial reduz o esforço auditivo quando palavras fonologicamente similares são separadas (WAGNER et al., 2016). 


$$
3 \text { Proposiçăo }
$$





\section{PROPOSIÇÃO}

Analisar o desempenho obtido na avaliação do esforço auditivo em indivíduos idosos com deficiência auditiva que sejam usuários de aparelho de amplificação sonora individual. 



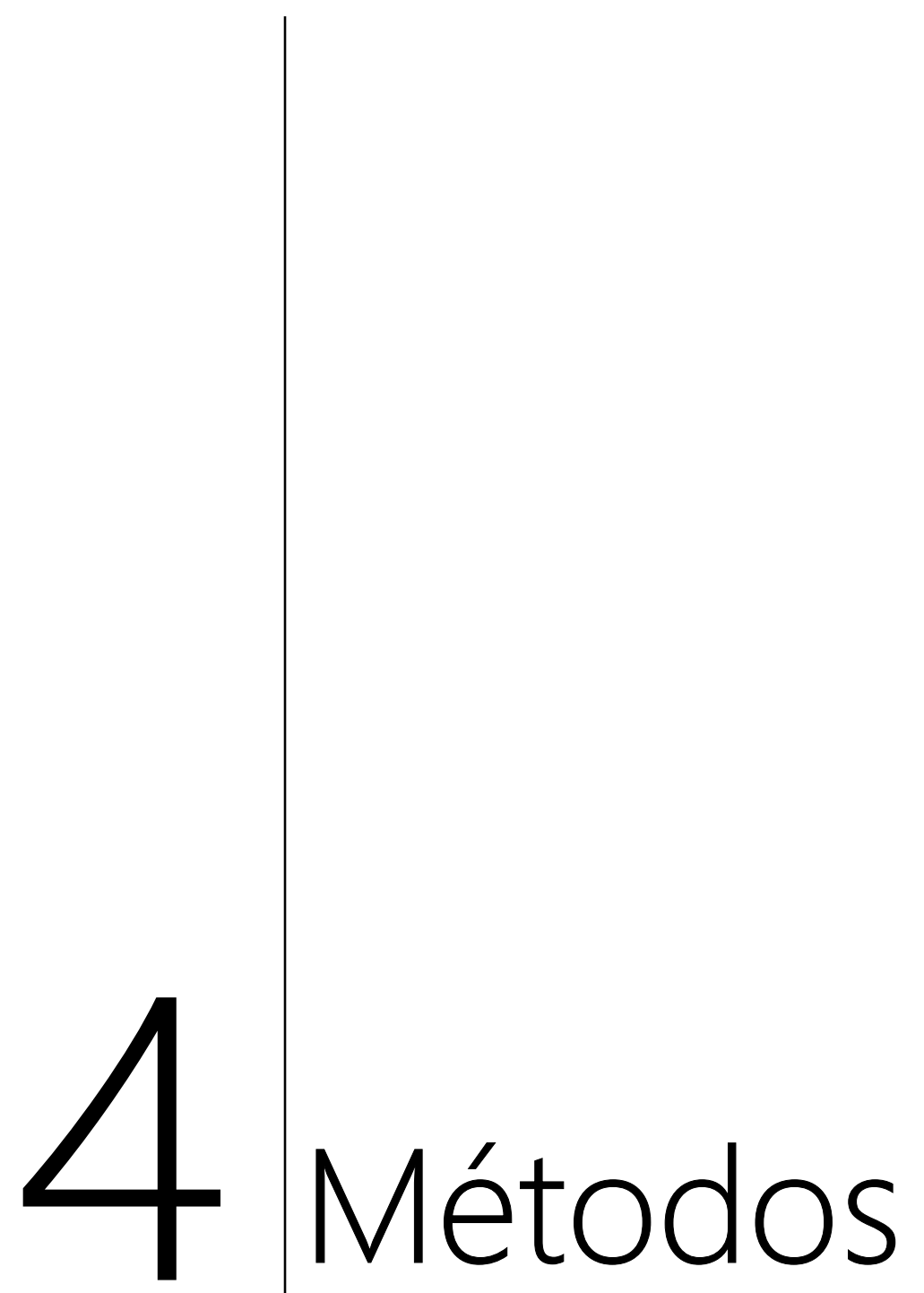





\section{MATERIA E MÉTODOS}

\subsection{ASPECTOS ÉTICOS}

O estudo foi aprovado pelo Comitê de Ética em Pesquisa da Faculdade de Odontologia de Bauru da Universidade de São Paulo (CEP/FOB/USP). Após entenderem e concordarem com os objetivos e procedimentos do estudo, todos os sujeitos da pesquisa assinaram o Termo de Consentimento Livre e Esclarecido (apêndice A), atestando sua permissão na participação e para publicação dos dados obtidos no estudo. O sigilo e a liberdade de se retirar do estudo a qualquer momento foram garantidos aos participantes.

O trabalho foi desenvolvido no Serviço de Saúde Auditiva da Clínica de Fonoaudiologia da Faculdade de Odontologia de Bauru (FOB/USP) em parceria com o Laboratório de Informação, Visão e Ação (LIVIA) do Departamento de Educação Física da Faculdade de Ciências da Universidade Estadual Paulista (Unesp campus de Bauru).

\subsection{DELIMITAÇÃO DA CASUÍSTICA}

Após a aprovação do CEP/FOB/USP, foi realizado levantamento dos prontuários de indivíduos matriculados na Clínica de Fonoaudiologia do Departamento de Fonoaudiologia da FOB/USP. Dos 297 prontuários analisados, 30 indivíduos foram selecionados e convocados para inclusão no estudo.

Desses 30 indivíduos, 4 não compareceram e 7 não apresentaram bom desempenho na avaliação de acuidade cognitiva e visual para aplicação do protocolo de avaliação do esforço auditivo. Ainda, não foi possível utilizar os dados coletados de 8 deles devido a falha técnica no momento do registro da dilatação da pupila. Dessa forma, foram selecionados 11 indivíduos para compor a amostra deste estudo.

Para a participação no estudo, os indivíduos deveriam seguir os seguintes critérios de inclusão: 
- Apresentar idade igual ou superior a 60 anos, independente do gênero, sendo, dessa forma, considerado idoso segundo os princípios da OMS (2002);

- Não apresentar alterações cognitivas e visuais;

- Apresentar diagnóstico de deficiência auditiva sensorioneural bilateral, de grau leve a severo (OMS, 2014), de acordo com a média quadritonal dos limiares audiométricos nas frequências de $500 \mathrm{~Hz}, 1000 \mathrm{~Hz}, 2000 \mathrm{~Hz}$ e $4000 \mathrm{~Hz}$. As orelhas foram consideradas simétricas quando apresentaram o mesmo grau e configuração de curva audiométrica, com diferença de até $15 \mathrm{~dB}$ entre as orelhas;

- Apresentar período de no mínimo seis meses com o uso de AASI binaural, seguindo as diretrizes do Instrutivo de Reabilitação Auditiva, Física, Intelectual e Visual do Ministério da Saúde (MINISTÉRIO DA SAÚDE, 2012);

- Apresentar acuidade visual normal ou corrigida com o uso de óculos ou lentes;

- Saber ler e escrever.

Os critérios abordados para exclusão do estudo foram:

- Ter realizado cirurgia otológica;

- Apresentar deformidades na orelha externa ou qualquer outra alteração que impeça a confecção de molde auricular, como cerúmen, hipertricose auricular, feridas ou descamações no canal auditivo;

- Apresentar outros comprometimentos (cognitivos, motores, neurológicos, psicológicos, visuais e/ou problemas graves de saúde);

- Não fazer uso efetivo do AASI.

Para garantir que os indivíduos atendessem os critérios de inclusão do estudo, inicialmente foram realizadas a avaliação cognitiva e a avaliação da acuidade visual pela fonoaudióloga pesquisadora responsável pelo estudo.

A avaliação da cognição foi realizada por meio do Montreal Cognitive Assessment (MoCA) (NASREDDINE et al., 2005), que é um instrumento de rastreio para deficiência cognitiva leve que foi desenvolvido e validado no Canadá e traduzido e adaptado para diversos idiomas. O MoCA acessa diferentes domínios cognitivos: atenção e concentração, funções executivas, memória, linguagem, habilidades visoconstrutivas, conceituação, cálculo e orientação. Seu tempo de aplicação foi de aproximadamente 10 minutos. O escore total é de 30 pontos, sendo considerado normal um escore de 26 ou mais. 
A acuidade visual foi medida com a tabela de Snellen (SALOMÃO, 2007), que pode ser aplicada por qualquer profissional da área da saúde devidamente capacitado (Ministério da Saúde, 2016). Essa avaliação considera a medida do menor ângulo formado entre os detalhes de um determinado objeto e sua imagem na retina, ou seja, a habilidade do sistema visual para discriminar detalhes. $O$ tipo de acuidade visual considerado foi o de reconhecimento, definido como a habilidade de identificar corretamente letras ou formas. A medição da acuidade visual com a tabela de Snellen é composta por letras de diferentes tamanhos dispostas em maiores, na parte superior, e em menores, na parte inferior.

Para a realização do teste, o indivíduo foi posicionado a uma distância de $1,80 \mathrm{~m}$ da figura (Figura 1) impressa em uma folha A4 e foi orientado a soletrar as letras conforme indicadas pelo aplicador do exame. Para determinar o resultado, foi adotada a notação de Snellen, que definiu a acuidade adulta normal como 20/20, ou seja, se o indivíduo soletrou corretamente até a linha oito, sua visão foi considerada dentro da normalidade. Foram utilizados corretores oculares conforme necessário (SALOMÃO, 2007).

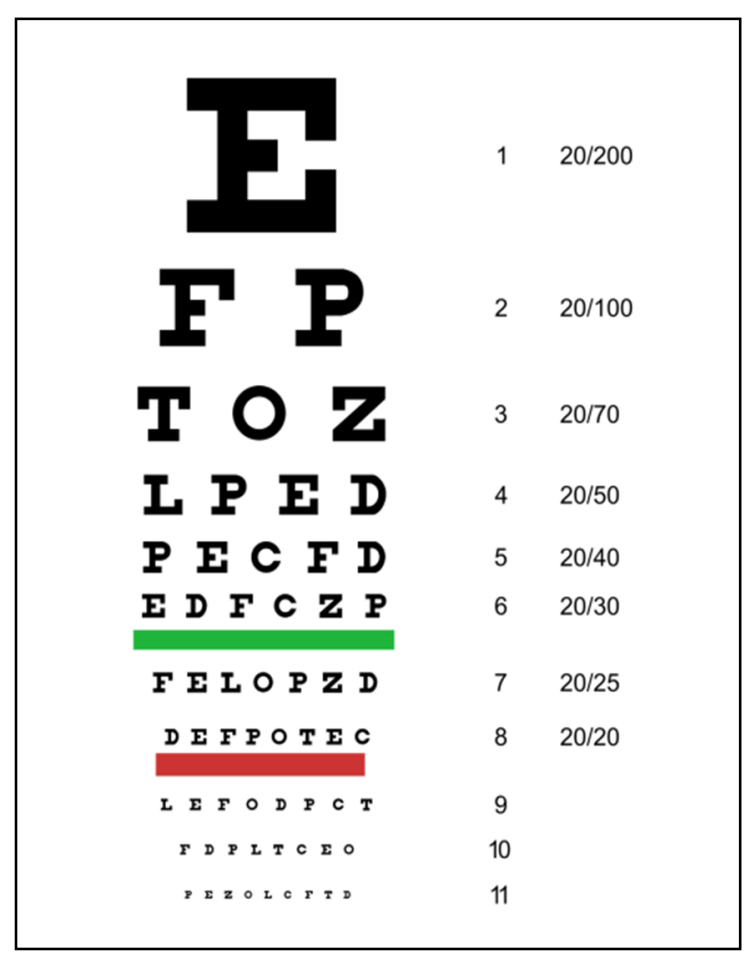

Figura 1. Tabela de Snellen 
Os participantes do estudo foram atendidos no Programa de Saúde Auditiva da Clínica de Fonoaudiologia do Departamento de Fonoaudiologia da FOB/USP. O serviço é credenciado no Ministério da Saúde para o atendimento de especialidade única (deficiência auditiva) desde 2004. As diretrizes para os atendimentos foram realizadas de acordo com o Instrutivo Saúde Auditiva ref. portaria GM 79 de 24 de abril de 2012, portaria GM 835 de 25 de abril de 2012 (diretrizes para tratamento e reabilitação/habilitação de pessoas com deficiência auditiva) e portaria GM/MS $n^{\circ} 2.073$, de 28 de setembro de 2004, que institui a política nacional de atenção à saúde auditiva.

\subsection{INSTRUMENTOS E PROCEDIMENTOS}

Após a seleção dos participantes, foi aplicado o protocolo do estudo para avaliação do esforço auditivo. A avaliação do esforço auditivo foi realizada por meio dos procedimentos de medida comportamental, avaliação da pupilometria com e sem uso de AASI, e também por meio de questionário, os quais estão descritos a seguir.

\subsubsection{MEDIDA COMPORAMENTAIL}

Para a avaliação da medida comportamental, foi utilizado o paradigma de dupla tarefa simples (PDT) com e sem o uso de AASI. Foi solicitado que o indivíduo realizasse duas tarefas, uma tarefa primária (TP) e uma tarefa secundária (TS). As tarefas foram apresentadas ao indivíduo sob três condições experimentais: em primeiro lugar, a TP administrada sozinha (condição da linha de base da TP); em seguida, a TS administrada sozinha (condição da linha de base da TS); e, por fim, as duas tarefas administradas simultaneamente (condição de dupla tarefa).

A TP constou do reconhecimento de percepção de fala na presença de ruído, e a TS de uma atividade de memorização. A orientação para a realização do PDT foi para que o indivíduo desse maior importância à TP sem deixar que a TS, neste momento apresentada simultaneamente à TP, interferisse em sua resposta. $O$ cálculo do esforço auditivo demandado foi realizado por meio da diferença de desempenho na TS e da condição de dupla tarefa. 
Para avaliação da percepção de fala, o procedimento foi a aplicação do Hearing in Noise Test (HINT-Brasil), cujo título em português é Avaliação da Percepção de Fala com Ruído, adaptado por Bevilacqua et al. (2008).

O HINT é constituído por 12 listas de sentenças com 20 sentenças em cada, totalizando 240 sentenças disponíveis. A intensidade de apresentação é variável até que seja estabelecido o limiar de reconhecimento de sentenças (LRF/HINT), que é obtido quando $50 \%$ das sentenças são repetidas corretamente. Após a apresentação da sentença para o participante, a resposta é aceita pelo avaliador quando a) todas as palavras forem repetidas corretamente, b) apenas os artigos definido e indefinido forem alterados e c) forem adicionadas palavras à sentença sem comprometer o sentido.

A técnica utilizada no HINT-Brasil é ascendente-decrescente (LEVIT; RIBER, 1967), e a intensidade das sentenças é adaptativa conforme a resposta do indivíduo. Os resultados são expostos em decibel (dB) pelos limiares de reconhecimento das sentenças, sendo positivo quando a fala está acima do ruído e negativo quando o som do ruído está acima do som de fala (WEISS; DEMPSEY, 2008).

A TS foi composta por uma tarefa de memória de cores denominada PALETA, a qual utiliza uma plataforma para auxiliar a execução de testes de dupla tarefa (CRUZ et al., 2019). O teste foi administrado em um tablet. Seguindo a mesma metodologia citada pela autora, após cada apresentação, o participante deveria lembrar e reproduzir uma sequência de duas cores. Por meio desta tarefa foi registrado o número de respostas corretas.

Inicialmente, os participantes receberam orientação de execução e de sessão de treino para garantir a forma correta da aplicação do protocolo do estudo. Foi explicado que eles realizariam três tarefas (TP, TS e PDT), sendo que a tarefa secundária e o paradigma de dupla tarefa seriam realizados sem e com o uso do aparelho auditivo.

\subsubsection{PUPILOMETRIA}

Foi realizada outra forma de avaliação do esforço auditivo por meio de uma avaliação fisiológica de monitoramento da dilatação da pupila denominada pupilometria. A avaliação da pupilometria proporciona mensurar a dilatação da pupila 
em situações de alteração cognitiva. Neste estudo, realizou-se simultaneamente a avaliação comportamental. Para esta avaliação, foi utilizado um eye tracker modelo Mobile Eye-5, da Applied Science Laboratories (ASL) (Figura 2), e a análise do movimento ocular foi realizada com os programas ASL Results Plus e MATLAB.

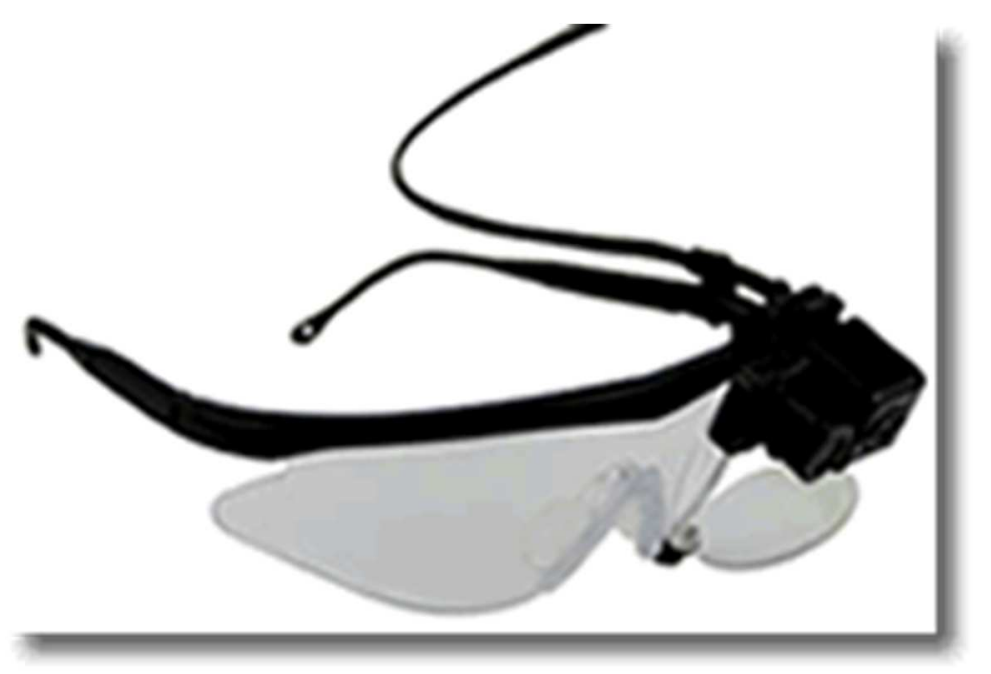

Figura 2. Eye tracker (Fonte: ASL EYE-TRAC® Series)

A fixação ocular foi identificada quando duas vezes o valor do desvio padrão da posição do olhar fosse inferior a $1^{\circ}$ do ângulo visual na horizontal e no eixo vertical por pelo menos 100 ms de duração da fixação (SIMIELI et al., 2017).

A duração média das fixações é a média de todas as durações de fixação em cada tentativa. A variabilidade das fixações foi calculada como a área da elipse de 85\%, cobrindo a posição das fixações (RODRIGUES et al., 2016).

Todos os dados foram gravados e interpretados em formato numérico. Para avaliação do esforço auditivo requerido, foram analisados os dados de dilatação média da pupila durante as tarefas.

A luminosidade do ambiente e da tela do tablet utilizado para a aplicação da PALETA foi controlada para minimizar os efeitos na dilatação da pupila, a qual permaneceu a mesma durante todas as condições.

\subsubsection{QUESTIONÁRIO}

O questionário utilizado foi o Speech, Spatial and Qualities of Hearing Scale (SSQ) (GATEHOUSE; NOBLE, 2004), o qual avalia as habilidades e as experiências que envolvem a audição em situações complexas de escuta do cotidiano. Além disso, 
inclui uma variedade de domínios, como situações de audição direcional relacionada a diferentes distâncias e ao movimento, segregação de sons e fluxos de vozes simultâneas, facilidade de escuta, naturalidade, clareza dos sons do cotidiano e de diferentes peças musicais e instrumentos. É composto por 49 questões divididas em três partes, sendo 14 relacionadas à audição para sons da fala, 17 que investigam diferentes componentes da audição espacial e 18 sobre as qualidades da audição. Os itens que especificamente visam o esforço auditivo incluem três perguntas da parte três, com questões que abordam a necessidade de concentração ao ouvir alguém ou algo, o esforço para ouvir o que está sendo dito na conversa com outros, e a capacidade de facilmente ignorar outros sons ao tentar ouvir algo, as quais foram analisadas.

\subsection{ANÁLISE DOS DADOS}

Os dados foram tratados e passaram por análise estatística no programa Statistical Package for the Social Sciences (SPSS). Nesse software, foi utilizado o teste t pareado nas comparações dos dados nas situações de tarefa primária (TP) com e sem o uso dos AASI, de tarefa secundária (TS) e de paradigma de dupla tarefa (PDT) sem e com o uso do AASI. No teste estatístico foi adotado nível de significância de $5 \%(p<0,05)$.

Os dados considerados para análise da TP (HINT) foram as médias da relação $S / R$, e os dados considerados para análise da TS foram os números de acertos em cada situação apresentada.

Para análise da pupilometria, foi utilizado o teste ANOVA de medidas repetidas, sendo adotado também o nível de significância de $5 \% \quad(p<0,05)$. Considerou-se a média da dilatação da pupila em um tempo de um segundo (s) que antecedeu o início da tarefa e o intervalo de tempo de início e término da aplicação de cada teste nas situações de TP e PDT sem e com o uso de AASI.

As questões do questionário Speech, Spatial and Qualities of Hearing Scale foram analisadas de forma descritiva. Os participantes foram orientados a responder as questões com base em suas experiências cotidianas com o uso do AASI. 



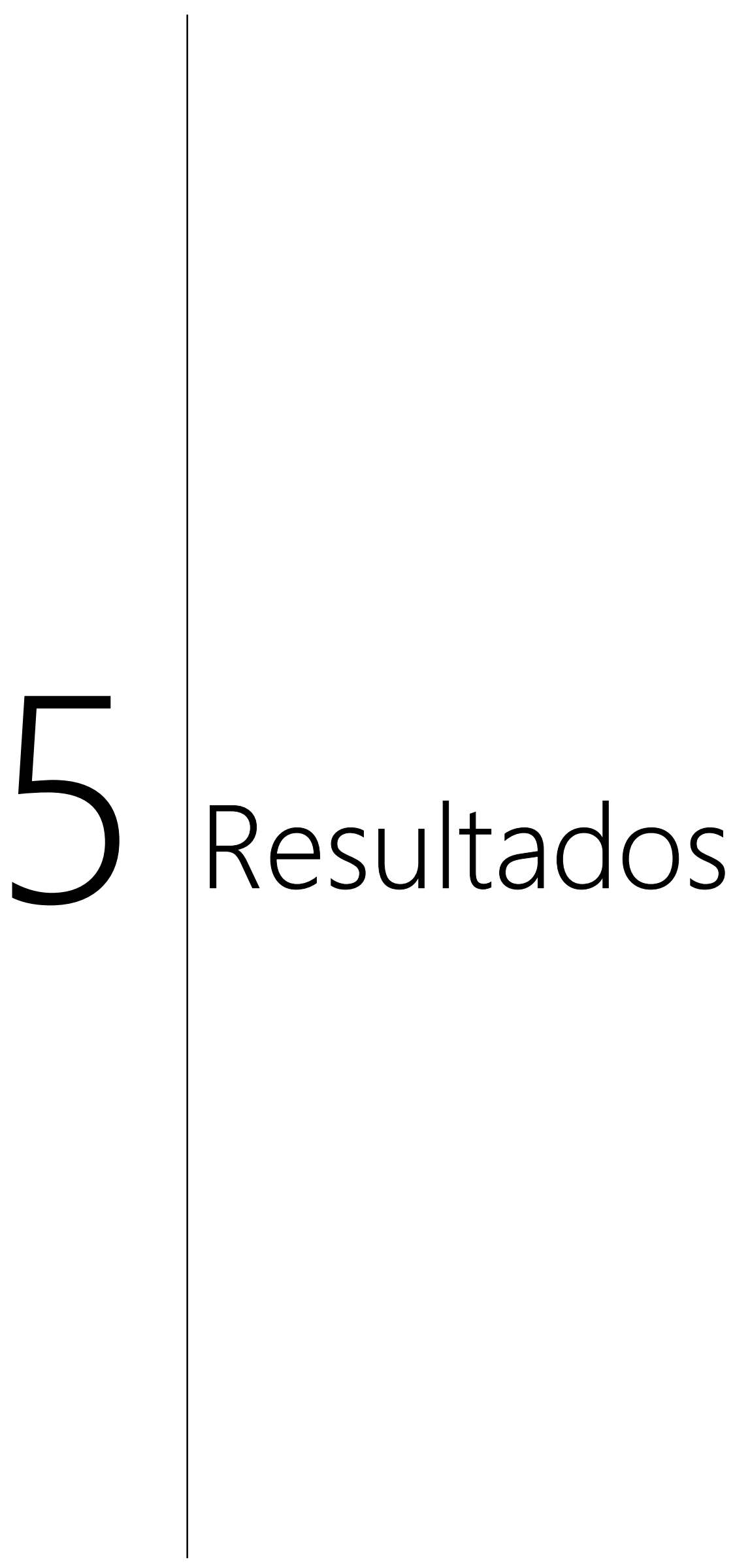





\section{RESULTADOS}

\subsection{CARACTERIZAÇÃO DA AMOSTRA}

A população do estudo foi composta por 11 indivíduos, em totalidade pela população idosa conforme especificado nos critérios de inclusão. Desses 11, cinco $(45,45 \%)$ são do gênero feminino e seis $(54,54 \%)$ são do gênero masculino, com idade entre 64 e 80 anos (média de 72,7 anos). Todos estavam adaptados com AASI bilateralmente e relataram fazer uso efetivo de ambos os aparelhos. A Tabela 1 apresenta os dados demográficos dos participantes.

Tabela 1 - Características dos participantes

\begin{tabular}{llll}
\hline Participantes (n=11) & Idade & \multicolumn{2}{c}{ Grau da perda auditiva } \\
\hline & & OD & OE \\
\cline { 2 - 3 } 1 & 75 & Severo & Severo \\
2 & 73 & Severo & Moderado \\
3 & 80 & Leve & Moderado \\
4 & 69 & Severo & Severo \\
5 & 70 & Leve & Leve \\
6 & 78 & Moderado & Moderado \\
7 & 64 & Moderado & Moderado \\
8 & 73 & Moderado & Moderado \\
9 & 77 & Moderado & Moderado \\
10 & 74 & Severo & Moderado \\
11 & 67 & Severo & Severo \\
\hline
\end{tabular}

Legenda: $\mathrm{n}=$ número de participantes

\subsection{ESFORÇO AUDITIVO}

\subsubsection{MEDIDA COMPORTAMENTAL}

Os resultados obtidos com o HINT na situação de TP mostraram diferença estatisticamente não significativa para os resultados do HINT na comparação dos resultados com o TP e no PDT com e sem o uso de AASI. Apesar de não haver 
diferença estatisticamente significativa, é possível observar que o desempenho dos participantes no teste HINT melhorou com o uso de AASI. A média da relação S/R obtida do HINT na situação de TP e PDT sem e com o uso do AASI está descrita no Gráfico 1:

Gráfico 1 - Comparação do HINT na TP e PDT sem e o com o uso do AASI

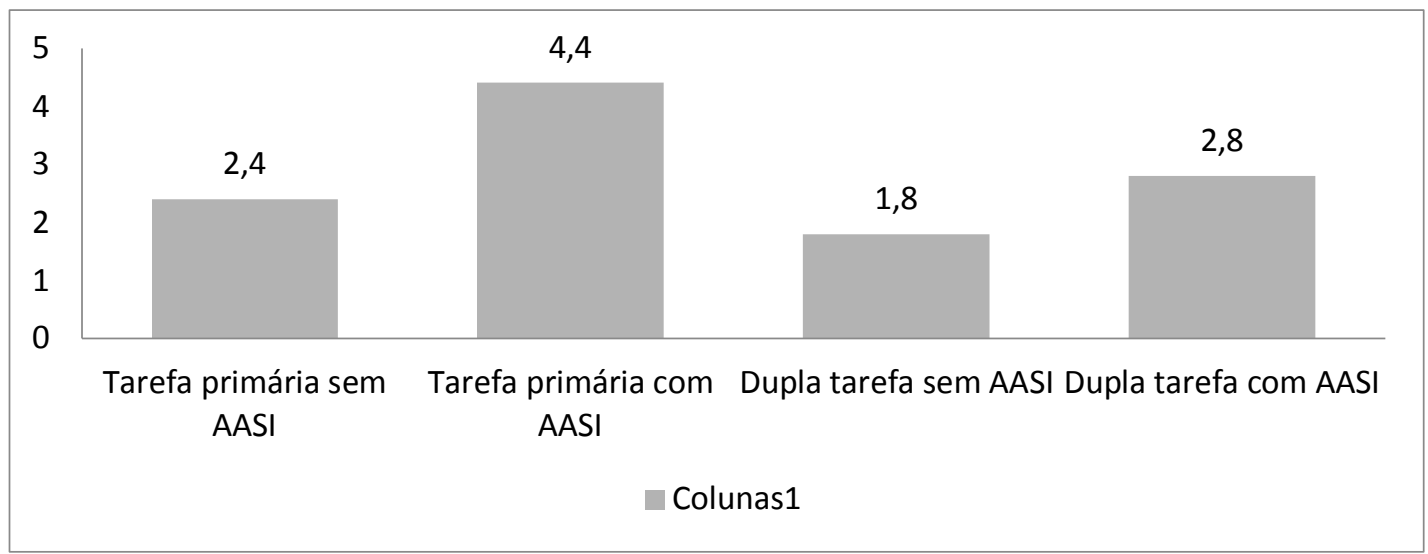

Legenda: HINT: Hearing in Noise Test; S/R: sinal/ruído; AASI: aparelho de amplificação sonora individual.

Na comparação do desempenho da TS aplicada separadamente e nas situações de PDT sem e com o uso do AASI, os resultados demonstraram que houve diferença estatisticamente significativa nas situações com e sem AASI. Isso mostra que o desempenho na realização da TS foi pior em situação de PDT, como descrito na Tabela 2:

Tabela 2 - Comparação da paleta na TS no PDT com e sem AASI.

\begin{tabular}{|c|c|c|c|c|}
\hline & Média & DP & $\mathbf{T}$ & $\mathbf{P}$ \\
\hline TS X PDT sem AASI & $-3,091$ & 3,015 & $-3,400$ & $0,007^{*}$ \\
\hline TS X no PDT com AASI & $-4,364$ & 2,873 & $-5,037$ & $0,001^{*}$ \\
\hline
\end{tabular}




\subsubsection{PUPILOMETRIA}

Conforme esperado, a variável dilatação média da pupila antes do início da tarefa $(M=128,4$ pixels, $E P=6,4)$, anterior ao início das condições experimentais, não foi significativamente afetada pelos efeitos da tarefa primária ou da dupla tarefa, nem pelo uso ou não do AASI, nem pela interação entre eles.

No entanto, de modo oposto ao esperado, durante as condições experimentais, a variável dilatação média da pupila foi significativamente menor durante o paradigma de dupla tarefa $(M=1,94$ pixels, $E P=0,69)$ do que durante a tarefa secundária $(M=6,70$ pixels, $E P=1,75)$. Ainda, a dilatação média da pupila $(F(1,10)=$ $11,36, p=0,007)$ não foi significativamente afetada pelo uso do aparelho, nem pela realização da tarefa primária ou do paradigma de dupla tarefa, nem pela interação entre eles.

\subsubsection{QUESTIONÁRIO}

Com relação à análise do questionário, para a descrição da percepção do esforço auditivo, foi utilizada uma escala de respostas de 0 a 10, em que se considerou como muito esforço as situações com respostas $\geq 5$. Foram aplicadas quatro questões do questionário SSQ em relação às situações do cotidiano com o uso de AASI. A disposição está descrita na tabela a seguir:

Tabela 3 - Análise das questões do questionário SSQ.

\begin{tabular}{lll}
\hline \multicolumn{1}{c}{ Questões } & MUITO & POUCO \\
\hline $\begin{array}{l}\text { Você tem que se concentrar muito quando está } \\
\text { escutando alguém ou alguma coisa? }\end{array}$ & $7(63,63 \%)$ & $4(36,36 \%)$ \\
\hline $\begin{array}{l}\text { Você tem que se esforçar muito para ouvir o que } \\
\text { está sendo dito em uma conversa? }\end{array}$ & $6(54,54 \%)$ & $5(45,45 \%)$ \\
\hline $\begin{array}{l}\text { Quando você é passageiro sentado ao lado do } \\
\text { motorista você consegue ouvir facilmente o que ele }\end{array}$ & $1(9,09 \%)$ & $10(90,90 \%)$ \\
$\begin{array}{l}\text { está falando? } \\
\text { Você consegue ignorar facilmente outros sons ao } \\
\text { tentar escutar alguma coisa? }\end{array}$ & $5(45,45 \%)$ & $6(54,54 \%)$ \\
\hline $\begin{array}{l}\text { Legenda: \%: porcentagem. } \\
\end{array}$
\end{tabular}



6 Discussão 



\section{DISCUSSÃO}

Com o aumento da expectativa de vida, a população tem se preocupado e, envelhecer com maior qualidade de vida. As consequências fisiológicas do envelhecimento podem trazer prejuízos quanto à audição, à cognição e aos aspectos sociais e emocionais do indivíduo (VIEIRA, 2004). Neste estudo, foi abordado o uso de AASI na população idosa e exposta a importância de se haver uma adequada forma de reabilitação para amenizar as consequências negativas da presbiacusia na comunicação do idoso.

O esforço auditivo refere-se à quantidade de recursos de processamento alocados a uma tarefa auditiva específica: quando as demandas da tarefa são altas, o indivíduo se esforça para alcançar um elevado nível de desempenho (GAGNÉ; BESSER; LEMKE, 2016). Sobretudo, o uso de dispositivos eletrônicos como o AASI em indivíduos com deficiência auditiva pode amenizar as consequências da perda auditiva, proporcionando auxílio para o entendimento da fala e percepção dos sons ambientais nos portadores de deficiência auditiva (BLASCA, 2006).

Foi possível observar nos resultados deste estudo que não houve diferença estatisticamente significativa no desempenho da TP sem e com o uso de AASI. Apesar desse achado, houve aumento expressivo na média da relação S/R no HINT quando realizada com e sem o uso do AASI nos participantes.

Pesquisadores relatam em seus achados científicos importantes alterações relacionadas ao envelhecimento que determinam perda da capacidade de adaptação do indivíduo ao meio ambiente, ocasionando maior vulnerabilidade e maior incidência de processos patológicos (PAPALEO NETTO, 2011). Os autores Russo e Almeida (1995) relataram que o desempenho da atividade de escuta na população idosa, como compreender a fala em ambientes com ruído, é uma tarefa complexa. Esta dificuldade não pode ser atribuída somente pelo tipo e pelo grau da perda auditiva, podem estar relacionadas a perdas da capacidade de realizar o processamento auditivo dos sons, associadas ao envelhecimento (VERSFELD, 2002). Mesmo com o uso do AASI, as queixas relacionadas à inteligibilidade de fala no ruído podem permanecer, queixas essas que podem ser justificadas por outros motivos. Um exemplo são os déficits no processamento cognitivo que ocorrem com o avanço da idade (TUN, O'KANE, 
WINGFIELD; 2002), os quais diminuem a sincronia neural e o processamento temporal dos sons (GORDON-SALANT, 2008). Esses fatores afetam diretamente a forma como a informação é organizada, fazendo com que o indivíduo interprete os sons de maneira mais lenta.

Dessa forma, apesar das dificuldades existentes devido à senilidade, a alternativa para amenizar os prejuízos da presbiacusia é o uso do AASI (MONDELLI; SOUZA, 2012; MARQUES; KOSLOWSKY; MARQUES, 2004). Se forem consideradas todas as características individuais, haverá uma boa aceitação da adaptação, gerando interação e, consequentemente, melhora da qualidade de vida do idoso com perda auditiva (CAMPOS; OLIVEIRA; BLASCA, 2010).

Quando o desempenho na tarefa secundária foi comparado na situação de dupla tarefa com e sem o uso do AASI, foi possível observar que houve piora no desempenho sem o AASI. Gagnè et al. (2017) afirmaram em seus estudos que o esforço auditivo é quantificado se há diminuição do desempenho da TS na situação de PDT. Assim, os achados deste estudo vão ao encontro das pesquisas realizadas pelos pesquisadores canadenses, pois os resultados também revelaram que houve maior esforço auditivo quando ocorreu a percepção de fala com ruído competitivo e uma tarefa de memória. Isso sugere que o PDT é uma forma eficaz de avaliar o esforço auditivo, e a PALETA é um instrumento que se mostrou adequado para ser utilizado como TS nessa forma de avaliação no idoso. Os achados deste estudo em relação à PALETA concordam com o descrito pelos autores Cruz et al., (2019).

Em complementação ao estudo, os dados analisados da pupilometria revelaram que as variáveis uso ou não do AASI não foram determinantes para que houvesse a alteração da dilação da pupila antes e durante a atividade proposta. Os achados desse estudo não concordam com o descrito na literatura pesquisada, cujos estudos mostram que a pupilometria reflete o esforço auditivo ao examinar como as mudanças no tamanho da pupila estão relacionadas a mudanças na atividade mental (KUCHINSKY; ECKERT; DUBNO, 2011) se adotado o AASI como um facilitador da audição para diminuir o esforço auditivo.

Assim como também não vão ao encontro dos achados dos autores Zekveld, Kramer e Festen (2010). Em seu estudo, sustentam que o esforço auditivo, conforme indicado pela resposta da pupila, aumenta com a diminuição da inteligibilidade da fala e indica que a pupilometria pode ser usada para examinar como os ouvintes atingem um determinado nível de desempenho em tarefa de escuta. 
Os autores McGarrigle et al. (2017) tiveram resultados semelhantes a essa pesquisa em relação à pupilometria em crianças, pois os dados não demonstraram significância para a mensuração do esforço auditivo quando o objetivo foi investigar o efeito da relação S/R no esforço auditivo (comportamental e pupilométrico) e relacioná-lo à fadiga (auto-relato e pupilometria).

Ao estudarem a pupilometria em idosos, Kuchinsky et al. (2013) concluíram que as análises da curva de crescimento revelaram que a resposta da pupila foi afetada pelo aumento do ruído que tornava a tarefa de percepção de fala mais difícil. Assim, sugerem que a pupilometria fornece outras evidências de dificuldade da tarefa além de apenas medidas comportamentais.

Como descrito anteriormente, as pesquisas mostram que a carga cognitiva pode estar diretamente ligada à alteração da dilatação da pupila. Porém, os resultados deste estudo mostram que não houve significância nas comparações na avaliação do esforço auditivo.

Isso sugere que, durante a aplicação do protocolo, mesmo com o treinamento e com as orientações aos participantes, os cuidados na aplicação e a máxima adequação do ambiente de teste, alguns fatores, como a falta da sustentação da atenção pelos participantes, a dificuldade na realização devido à complexidade da tarefa e até mesmo o número da amostra do estudo, podem ter influenciado os resultados apresentados.

Neste estudo, a análise das questões aplicadas do questionário Speech, Spatial and Qualities of Hearing Scale (SSQ) mostra que, quando a questão foi pontual quanto ao esforço e à concentração, o relato da maioria dos participantes (questão de esforço $=54,54 \%$ e concentração=63,63\%) mostrou elevado nível de esforço. Porém, em relação às questões sobre ouvir e ignorar ruído de fundo, os relatos mostraram pouca dificuldade. Esses resultados demonstram a importância do questionário como complementação da avaliação proposta. No entanto, ainda é incipiente em relação à determinante da avaliação do esforço auditivo.

Em todo o contexto do estudo realizado houve uma grande dificuldade para composição da amostra. Foram necessárias várias análises com determinações específicas para a elaboração de um protocolo adequadamente sensível a população idosa, visto que os critérios de exclusão abrangeram achados típicos de alterações senis, como alteração visual/cognitiva, que poderiam influenciar no desempenho durante as atividades propostas. Além disso, houve dificuldade no registro dos dados 
da pupila pelas características anatômicas encontradas, como as alterações palpebrais.

A ausência de um projeto piloto para ajustar a complexidade da tarefa e a movimentação do indivíduo durante a realização das tarefas pode ter influenciado o registro da pupilometria para confirmação da dilatação aumentada durante maior esforço auditivo durante as tarefas.

Assim, apesar de os resultados encontrados não serem estatisticamente significativos, é importante ressaltar que os dados podem contribuir para o aperfeiçoamento da avaliação do esforço auditivo na população idosa. Isso fica claro ao se levar em conta que, atualmente, a adaptação do aparelho auditivo é baseada no protocolo padronizado de seleção e de adaptação em adultos (AMERICAN ACADEMY OF AUDIOLOGY, 2008). Isso é crucial, pois a população idosa tem características peculiares que devem ser consideradas para que haja a adequada reabilitação dos indivíduos com perda auditiva nessa faixa etária (ROEDAS et al., 2014; BLASCA et al., 2014; BERTOZZO, 2017). 


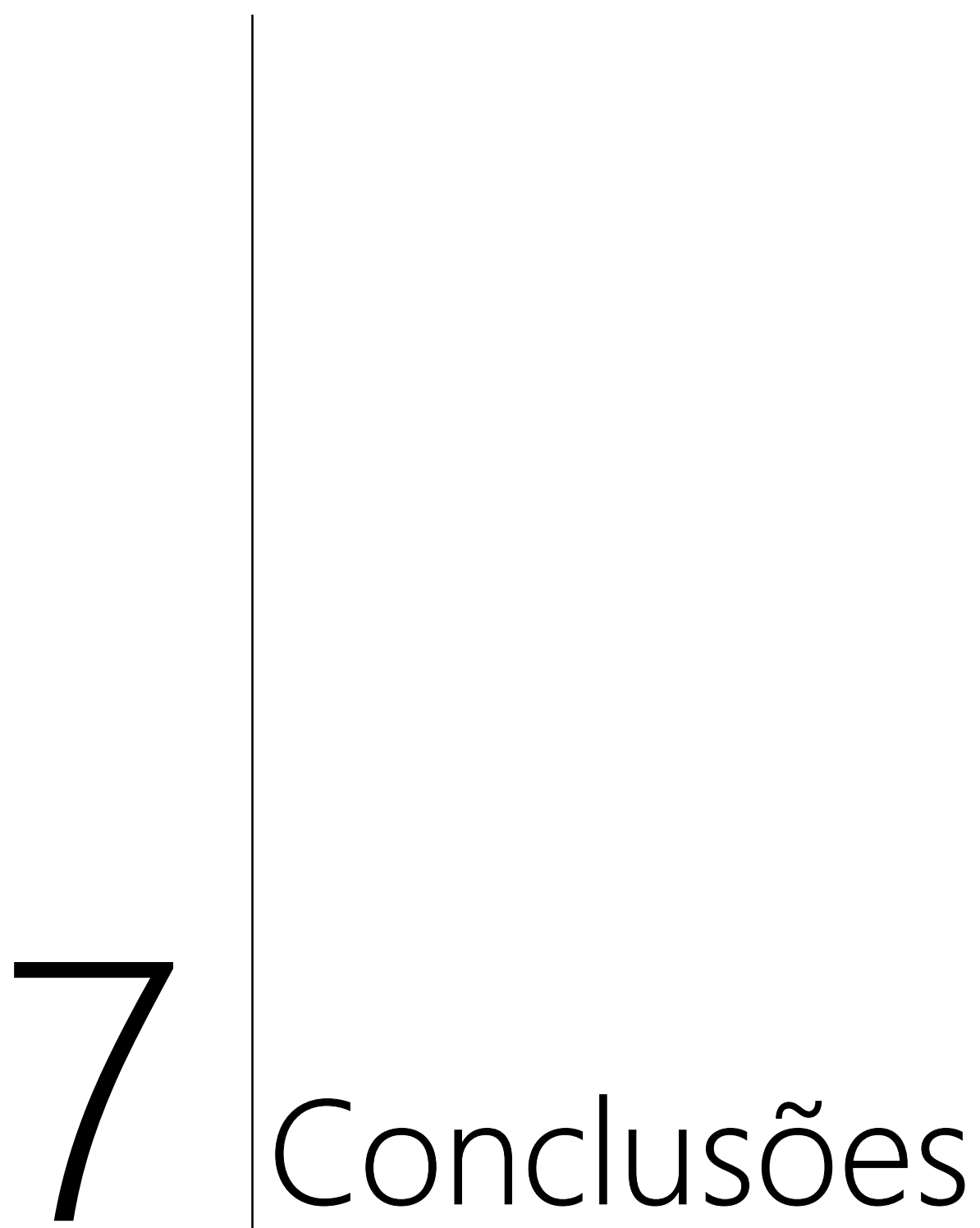





\section{CONCLUSÕES}

A partir do estudo com o objetivo de analisar o desempenho obtido na avaliação do esforço auditivo em indivíduos idosos que possuem deficiência auditiva e que são usuários de aparelho de amplificação sonora individual, foi possível concluir que:

- Houve esforço auditivo nas situações de tarefa primária e de dupla tarefa com e sem o uso do AASI;

- O uso do AASI não foi um fator significativo para melhorar o desempenho nas tarefas de escuta nem para diminuir o esforço auditivo, porém houve melhora expressiva no desempenho dos participantes com o uso do AASI; 

Referências 



\section{REFERÊNCIAS}

Academia Americana de Audiologia. Guidelines for theaudiologic management oh adulthearingimpairment. $2008 . \quad$ Disponível em: http://www.audiology.org/resources/documentlibary/Documents/haguidelines.pdf.

ASHA. American Speech-Language_ HearingAssociation. Guidelines for hearingaldfitting for adults [Guidelines]. 1998. Disponível em: <http:/www.asha.org/policy>. Acesso em 10 fev. 2011. [Acesso em: 09 Jan.2020].

BALTES, P.B.; LINDENBERGER, U. Emergence of a powerful connection between sensory and cognitive functions across the adult life span: A new window to the study of cognitive aging? PsycholAging v.12, p.12-21, 1997.

BERTOZZO, M.C. Estudo comparativo dos métodos prescritivos NAL-NL2 e DSLv5.0 na programação individualizada do AASI para o idoso. 2017. Dissertação de Mestrado - Faculdade de Odontologia de Bauru. Universidade de São Paulo, Bauru.

BESS, F.H.; HEDLEY-WILLIAMS, A.; LICHTENSTEIN, M.J. Avaliação audiológica dos idosos. In: MUSIEK, J.; RITELANAM, W.F. Perspectivas Atuais em avaliação Auditiva. 1. ed. Barueri> Editora Manole. 2001. Cap.12. p. 343 - 367.

BEVILACQUA, M.C et al. The Brazilian Portuguese Hearing In Noise Test. Int J Audiol, Hamilton: v. 47, n. 6, 2008 p. 364-365.

BLASCA, W. Q.; FERRARI, D. V.; JACOB, R. T. S. Dispositivos eletrônicos aplicados a surdez: conceitos básicos. In $O$ processo de comunicação: contribuição para a formação de professores na inclusão de indivíduos com necessidades educacionais especiais. Pulso Editorial, 2006.

BLASCA, W.Q. et al. ImportanceoftheRecd in theFittingofHearing Aids in ElderlyAudiology Now.2014.

BOURLAND-HICKS, C.; THARPE, A. M. Listening effort and fatigue in schoolage children with and without hearing loss. J Speech Lang Hear Res, v. 45, p. 573-584, 2002.

CAMPOS, K.; OLIVEIRA, J.R.M.; BLASCA, W.Q. Processo de adaptação de aparelho de amplificação sonora individual: elaboração de um DVD para auxiliar a orientação a indivíduos idosos. v.15, n.1, p.19-25, 2010. 
CARMO, L.C.; SILVEIRA, J.A.M.; MARONE, S.A.M.; D'OTTAVIANO, F.G.; ZAGATI, L.L.; LINS, E.M.D.S. Estudo audiológico de uma população idosa brasileira. Revista Brasileira de Otorrinolaringologia. v.74, n.3, p.342-349, 2008.

CHABA. WorkingGroupon speech UnderstandingandAging. Speech understandingandaging. J AcoustSoc Am. 1988; (3): 859-95.

CRUZ, A.D.; GAGNÉ, J.P.; CRUZ, W. M.; ISOTANI, S.; GAUTHIER-COSSETTE, L.; JACOB, R.T.S. The effects of using hearing aids and a frequency modulated system on listening effort among adolescents with hearing loss. International Journal of Audiology, n. 9, p.1-7, 2019.

Dawes, P.; Emsley R.; Cruickshanks KJ, et. al. Hearing Lossand Cognition: The Role of Hearinga Aids, Social Isolation and Depression, 2015. Disponível em:<https://doi.org/10.1371/journal.pone.0119616. [Acesso em: 2 dez 2019]

FRASER, S. et al. Evaluating the effort expended to understand speech in noise using a dual-task paradigm: The effects of providing visual speech-cues. J Speech Lang Hear Res, v. 53, n. 1, p. 18-33, 2010.

GAGNÉ, J.P.; BESSER, J.; LEMKE, U. Behavioral assessment of listening effort using a dual-task paradigm: a review. Trends in Hearing. v.21, p.1-25, 2017.

GAGNÉ, J.P.; BESSER, J.; LEMKE, U. Behavioral Assessment of Listening Effort Using a Dual-Task Paradigm: A Review. TrendsHear, v.21, p.1-25, 2016.

GALLACHER, J.; ILUBAERA, V.; BEN-SHLOMO, Y.; BAYER, A.; FISH, M., BABISCH, W.; ELWOOD, P. Auditory threshold, phonologic demand, and incident dementia. Neurology. v.79, n.15, p.1583-90, 2012.

GATEHOUSE, S.; NOBLE, W. The Speech, Spatial and Qualities of Hearing Scale (SSQ). Int J Audiol, v. 43, p. 85-99, 2004.

GIURANI, F.; HASAN, M. Abuse in elderly people: the Granny Battering revisited. Arch Gerontol Geriatr. v.31, n.3, p.215-20, 2000.

GOLDWATER, B. C. Psychological significance of pupillary movements. Psychol Bull. v.77, n.5, p.340-55, 1972 
GRANHOLM, E.; VERNEY, S. Pupillary responses and attentional allocation problems on the backward masking task in schizophrenia. International Journal of Psychophysiology, n.52, p.37-51, 2004.

HEINRICH, A.; HENSHAW, H.; FERGUSON, M.A. Only behavior al but not self-report measures of speech perception correlate with cognitive abilities. Front Psychol. v.5, p.576-577, 2016.

HUMES, L.E.; COUGHLIN, M. Aided speech-identification performance in single-talker competition by older adults with impaired hearing. Scand J Psychol. v.50, n.5, p.48594, 2009.

IBGE. Projeção da População do Brasil por Sexo e Idade - 1980-2050 - Revisão 2008, v. 24. Rio de Janeiro: IBGE,2008.

INSTRUTICO SAUDE AUDITIVA (ISA). Diretrizes para tratamento e reabilitação/habilitação de pessoas com deficiência auditiva. Ref. Portária GM 79 de abril de 2012 e Portaria GM 835 de 25 de abril de 2012.

KIM, T.S.; CHUNG, J.W. Evaluationof age-relatedhearingloss. Korean J Audiol. v.17, n.2, p.50-3, 2013.

KUCHINSKY, S.E.; ECKERT, M. A.; DUBNO, J. R. The eyes are the windows to the ears: Pupil size reflects listening effort. Audiology Today, n.23, p.56-59, 2011.

KUCHINSKY, S.E.; AHLSTROM, J.B.; KENNETH, I.V.; J.R., CUTE, S, L.; HUMES, L.E.; DUBNO, J.R.; ECKERT, M. A. Pupilsize varies withwordlisteningand response selectiondifficulty in olderadultswithhearingloss. Psychophysiology. v.50, n.1, p.23-34, 2013.

KUCHINSKY, S.E.; KENNETH, I.V.; AHLSTROM, J.B.; J.R., CUTE, S, L.; HUMES, L.E.; DUBNO, J.R.; ECKERT, M. A. Task-related vigilance during speech recognition in noise for older adults with hearing loss IN Exp Aging Res. v.42, n.1, p.64-85, 2016.

LARSBY, B.; HALLGREN, M.; LYXELL, B. Cognitive performance and perceived effort in speech processing tasks; Effects of different noise backgrounds in normal-hearing and hearing-impaired subjects. Int J Audiol, v. 44, p. 131-143, 2005.

LEVITT, H.; RABINER, L.R. Userof a sequentialstrategy in intelligibilitytesting. J acoustSoc Am. v.42, n.3, p. 609- 12, 1967. 
LIMA, A.P.; DELGADO, E.I. A melhor idade do brasil: aspectos biopsicossociais decorrentes do processo de envelhecimento. Ulbra e Movimento (REFUM). v.1, n.2, p.76-91, 2010

LIN, F.R.; THORPE, R.; GORDON-SALANT, S.; FERRUCCI, L. Hearing loss prevalence and risk factors among older adults in the United States. Journal of Gerontology. Series A, Biological Sciences and Medical Sciences, v.66a , n.5, p.582590, 2011.

LINDENBERGER, U.; BALTES, P. Sensoryfunctioningandintelligence in old age: A strong connection. PsychologyofAging, v.9, p.339-355, 1994.

MARINI, A.L.S.; HALPERN, R.; AERTS, D. Sensibilidade, especificidade e valor preditivo da queixa auditiva. Revista de Saúde Pública. v.39,n.6, p.982-984, 2005.

MARQUES, A.C.O.; KOZLOWSKI, L.; MARQUES, J.M. Reabilitação auditiva no idoso. Revista Brasileira de Otorrinolaringologia, 70(6), 806-811, 2004.

MCGARRIGLE, R.; MUNRO, K.J.; DAWES, P.; STEWART, A.J.; MOORE, D.R.; BARRY, J.G. Listening effort and fatigue: what exacty are we measuring? A British Society of Audiology Cognition in Hearing Special Interest Group "whitepaper". Int J Audiol. v.53, n.7, p.433-40, 2014

MCMAHON, C.M.; BOISVERT, I.; DE LISSA, P.; GRANGER. L.; IBRAHIM. R.; LO, C.Y. Monitoring alpha oscillation sand pupil dilation across a performance-intensity function. Front. Psychol. v.7, n.74, p.1-12, 2016.

MILLS, T.L. Comorbid depressive symptomatology: isolating the effects of chronic medical conditions on self-reported depressive symptoms among community-dwelling older adults. Soc Sci Med. v.53, n.5, p.569-78, 2001.

Ministério da Saúde. Portárian ${ }^{2}$.073, de 28 de setembro de 2004. Institui a política nacional de atenção à Saúde Auditiva. 2004. [acesso em 2019 jan.9] Disponível em: http://bvsms.saude.gov.br/bvs/saudelegis/gm/2004/prt2073 2809 2004.html

MONDELLI, M.F.C.; SOUZA, P.J.S. Qualidade de vida em idosos antes e após a adaptação do AASI. BrazilianJournalofOtorhinolaryngology, v.78, n.3, p.49-56, 2012.

MURPHY, D.R.; DANEMAN, M.; SCHNEIDER, B.A. Why do older adults have difficulty following convesations? Psychol Aging. v.2, n.1, p.49-61, 2006 
PALS, C.; SARAMPALIS, A.; BAŞKENT, D. Listening effort with cochlear implant simulations. J Speech Lang Hear Res. v.56, n.4, p.1075-84, 2013.

PAPALEO-NETTO, M. O estudo da velhice: histórico, definição do campo e termos básicos. In: Freitas EV, Py L, Cançado FAX Doll J, Gorzini ML. Tratado de geriatria e gerontologia. Rio de janeiro: Guanabara Koogan, 2011. p.3-13

PICHORA-FULLER, K.; SINGH, G. Effects of age on auditory and cognitive processing; implications for hearing aid fitting and audiologic rehabilitation. Trends Amplif, v.10, n.1, p.29-59, 2006.

PICHORA-FULLER, M.; SOUZA, P. Effects of aging on auditory processing of speech. Int J Audiol. v.42, n.2, p. 6-11, 2003.

PICHORRA-FULLER, M.K. Cognitive aging and auditory information processing. IJA. v.42, n.2, p.S26 - S32, 2003.

REIS, R.R.; ESCADA, P. Presbycusis: do we have a third ear? Braz J Otorhinolaryngol. v.82, n.6, p.710-714, 2016.

RODRIGUES, S.T.; POLASTRI, P.F.; GOTARDI, G.C.; AGUIAR, S.A.; MESAROS, M.R.; PESTANA, M.B.; BARBIERI, F.A. Postural control during cascade ball juggling: effects of expertise and base of support. Percept. Mot. Skills. v.123, p. 279-294, 2016.

ROEDAS, A.P. A análise da correlação da alteração da orelhaexternaemmedidas in situ CoDas. v.26, n.2, abr 2014.

RONNBERG, J. et al. The ease of language understanding (ELU) model: Theoretical, empirical, and clinical advances. Front SystNeurosci, v. 7, n. 31, 2013.

RUSSO, I.C.P.; ALMEIDA, K.; FREIRE, K.G.M. (2003). Seleção e adaptação da prótese auditiva para o idoso. In: Almeida, K.; LÓRIO, M.C.M. Próteses auditivas: fundamentos teóricos e aplicações clínicas. São Paulo (SP): Lovise.

SANTANA, C. Esforço para ouvir e envelhecimento auditivo: Análise de relações funcionais entre idade, sensibilidade auditiva e atenção dividida. Tese. Universidade de Brasília, 2017.

SALOMÃO, S.R. Desenvolvimento da acuidade visual de grades. Psicol USP, v.18, n.2, p. 63-81, 2007. 
SCHOEVERS, R.A.; BEEKMAN, A.T.,; DEEG, D.J.; GEERLINGS, M.I.; JONKER, C.; VAN TILBURG, W. Risk factors for depression in later life; results of a prospective community based study (AMSTEL). J Affect Disord. v.59, n.2, p.127-37, 2000.

SIMIELI, L.; VITÓRIO, R.; RODRIGUES, S.T.; ZAGO, P.F.P.; IGNACIO PEREIRA, V.A.; BAPTISTA, A.M.; DE PAULA, P.H.A.; PENEDO, T.; ALMEIDA, Q.J.; BARBIERI, F.A. Gaze and motor behavior of people with PD during obstacle circumvention. Gait Posture n.58, p.504-509, 2017.

SCIALFA, C. The role of sensory factors in cognitive agin gresearch. Canadian Journal of Experimental Psychology, v.56, n.3, 153-163, 2002.

STEWART, R., WINGFIELD, A. Hearing loss and cognitive effort in older adults' report accuracy for verbal materials. J Am Acad Audiol. v. 20, n. 2, p.147-54, 2009.

TUN, P.; BENICHOV, J.; WINGFIELD, A. Effortful processing of spoken sentences in younger and older adults: Effects of age and hearing. Paper presente dat the Cognitive Aging Conference. 2008.

TUN, P.A.; MCCOY, S.; WINGFIELD, A. Aging, hearing acuity, and the attentional costs of effortful listening. Psychol Aging. v.24, n.3, p.761-6, 2009

VALENTE, M. et al. Guidelines for the Audiologic management of adult hearing impairment. American Academy of Audiology. Audiology Today, v.18, n. 5, p.1-44, 2006.

VERSFELD, N.J.; DRESCHLER, W.A. The relationship between the intelligibility of time-compressed speech and speech in noise in young and elderly listeners. J Acoust Soc Am. v.111, n.1, p.401-8, 2002.

VIEIRA, E.B. Manual de gerontologia: um guia teórico-prático para profissionais, cuidadores e familiares. $2^{\mathrm{a}}$ ed. Rio de Janeiro: Revinter, 2004.

WAGNER, A.; PALS, C.; BLECOURT, C.M.; SARAMPALIS, A.; BAŞKENT, D. Does signal degradation affect top-down processing of speech? v.894, p.297-306, 2016.

WAGNER, A.E.; TOFFANIN, P.; BASKENT, D. The timing and effort of lexical access in natural and degraded speech. Front. Psychol. v.7, n.398, p.1-14, 2016. 
WEISS, D.; DEMPSEY, J.J. Performance of bilingual speakrson the English and Spanish versions of the Hearing in Noise Test (HINT). J Am Acad Audiol. v.19, n.1, p.5-17, 2008.

WORRALL, L.E.; HICKSON, L.M. Communication disability in aging from prevention to intervention. New York: Thomson, Delmar Learning, 2003.

WU, Y.H. et al. Measuring listening effort: driving simulator versus simple dual-task paradigm. Ear Hear, v. 35, n.6, p. 623-32, 2014.

ZAZOVE, P.; MEADOR, H.E.; AIKENS, J.E.; NEASE, D.E; GORENFLO, D.W. Assessment of depressive symptoms in deaf persons. J Am Board Fam Med. v.19, n.2, p.141-7, 2006.

ZEKVELD, A.A.; KRAMER, S.E.; FESTEN, J.M. Pupil response as an indication of effortful listening: the influence of sentence intelligibility. Ear Hear. v.31, n.4, p.480-90, 2010.

MOORE, D.R.; EDMONDSON-JONES, M.; DAWES, P.; FORTNUM, $H_{.}$; MCCORMACK, A.; PIERZYCKI, R.H.; MUNRO, k.j. Relation between Speech-inNoise Threshold, Hearing Loss and Cognition from 40-69 Years of Age. PLoS ONE v.9, n.9, e107720, 2014.

GUIJO, L. M.; HORIUTI, M.B.; NARDEZ, T.M.B.; CARDOSO A.C.V. Listening effort and working memory capacity in hearing impaired individuals: an integrative literature review.Rev. CEFAC. v.20, n.6, p.798-807, 2018

NASREDDINE, Z.S.; PHILLIPS, N.A; BÉDIRIAN, V.; CHARBONNEAU, S.; WHITEHEAD, V.; COLLIN, I.; CUMMINGS, J.L.; CHERTKOW, H. The Montreal Cognitive Assessment, MoCA: a brief screening tool for mild cognitive impairment. J Am Geriatr Soc. v.53, n.4, .695-9, 2005. 

Anexos 

Anexo A: Avaliação Cognitiva de Montreal - Montreal CognitiveAssessment (MoCA)

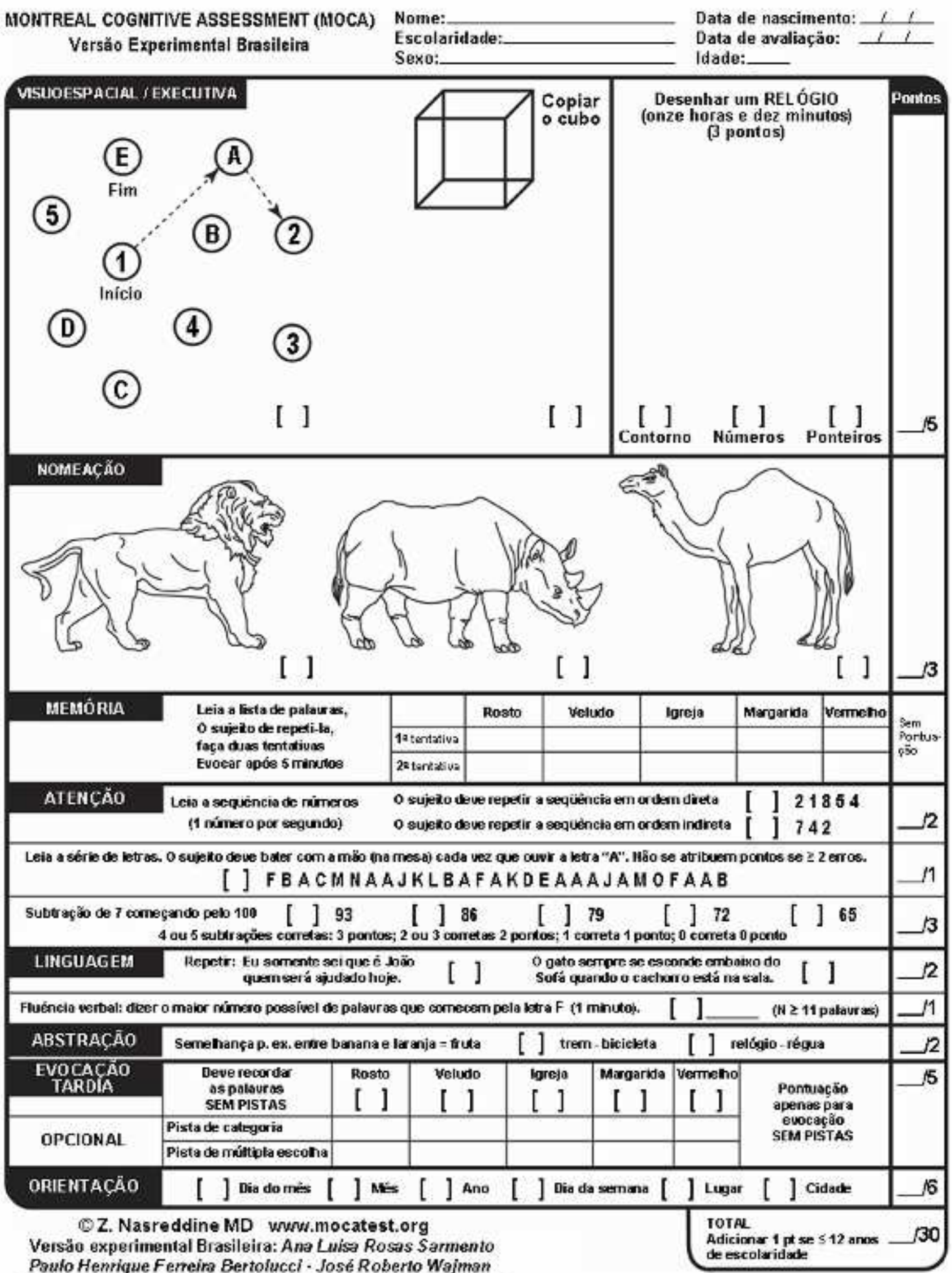

(UNIFESP.SP 2007) 
Anexo B:Termo Consentimento Livre Esclarecido (TCLE).

\section{TERMO DE CONSENTIMENTO LIVRE E ESCLARECIDO}

Venho por meio deste documento convidá-lo(a) sob seu consentimento a participar de uma pesquisa com o título: "O esforço auditivo com uso da amplificação por meio de paradigma de dupla tarefa e pupilometria", que tem como objetivo analisar o desempenho obtido na avaliação do esforço auditivo em indivíduos idosos com deficiência auditiva com e sem o uso de aparelho de amplificação sonora individual (AASI) utilizando paradigma de duas tarefas e pupilometria.

$\mathrm{O}(\mathrm{A})$ senhor(a) foi comunicado a comparecer à Clínica de Fonoaudiologia da Faculdade de Odontologia de Bauru para realização dos seguintes procedimentos:

- Questionário, no qual o(a) senhor(a) deverá responder perguntas que envolvem a audição em situações complexas de escuta do cotidiano;

- Avaliação da acuidade visual, na qual o(a) senhor(a) deverá permanecer sentado(a) em uma cadeira posicionada a 3 metros da parede onde haverá uma escala de letras, e após visualizá-las o (a) senhor(a) deverá identificar as letras listadas;

- Avaliação cognitiva, a qual contém tarefas simples que acessa diferentes domínios cognitivos: atenção e concentração, funções executivas, memória, linguagem, habilidades viso-construtivas, conceituação, cálculo e orientação;

- Repetição de listas de sentenças apresentadas em campo livre; e memorização de cores por meio de um aplicativo de paleta de cores presente em um tablet. Esses procedimentos serão realizados separadamente e de forma simultânea com e sem o uso dos AASI. Durante esses testes haverá o monitoramento da dilatação da pupila através de um equipamento portátil posicionado na cabeça. Estes testes poderão causar desconforto em decorrência do tempo levado para a aplicação, porém o nível de apresentação das frases não será desconfortável.

A realização dos procedimentos específicos da pesquisa levará em torno de uma hora e meia e serão acompanhados por profissionais capacitados. Estes procedimentos não são invasivos, mas por abordarem diretamente a deficiência auditiva podem causar constrangimento ou lembranças de experiências ou situações vividas que causem sofrimento psíquico, físico, intelectual, social, cultural ou espiritual, além de desconforto pelo tempo exigido. $\mathrm{O}(\mathrm{A})$ senhor(a) será orientado(a) a informar ao profissional caso sinta algum incômodo de qualquer natureza para que seja modificada a abordagem, e caso haja desconforto devido a duração dos procedimentos o(a) senhor(a) poderá solicitar um tempo para descanso. Os procedimentos a serem realizados servem para avaliar o nível de esforço auditivo necessário para realizar tarefas de audição ao mesmo tempo em que o(a) senhor(a) realiza outras tarefas, e demonstrará o quanto o uso do seu AASI o(a) auxilia nestas situações.

Espera-se que os dados obtidos nesta pesquisa auxiliem no direcionamento da adaptação do AASI e reabilitação auditiva de indivíduos idosos no que se refere ao esforço auditivo demandado por essa população quando apresentando deficiência auditiva, auxiliando assim na melhoria da qualidade de vida dos mesmos. Os resultados dos procedimentos com os indivíduos participantes, já usuários de AASI, demonstrarão o esforço auditivo requerido pelos mesmos ao realizar outras tarefas 
simultaneamente, e tais dados contribuirão na orientação de estratégias de comunicação e reabilitação auditiva com a amplificação.

Neste estudo o(a) senhor(a) não pertencerá a nenhum grupo, sendo avaliado de maneira individual e analisado e comparado somente com o seu próprio desempenho com e sem o uso dos AASI. Seu comparecimento para o estudo não oferece nenhum custo ou risco. Os custos para o seu transporte até a Clínica de Fonoaudiologia da FOB-USP e alimentação serão por sua conta e caso necessário haverá ressarcimento das despesas pela sua participação nesta pesquisa. Caso Ihe ocorra algum dano decorrente de sua participação nesta pesquisa, o(a) senhor(a) será indenizado. A sua participação na pesquisa não trará nenhum gasto financeiro nem trará riscos à sua dignidade, mas o(a) senhor(a) deverá ficar na clínica aproximadamente umahora e meia para a aplicação dos testes. Se houver algum dano decorrente de sua participação na pesquisa será garantido o direito à indenização.

Durante ou posteriormente a pesquisa, o(a) senhor(a) tem a garantia de receber resposta a perguntas ou esclarecimento a qualquer dúvida sobre os procedimentos, riscos, benefícios e outros assuntos relacionados à pesquisa, além dos AASI que serão adaptados de acordo com a disponibilidade e a necessidade. Sua participação é voluntária e você pode retirar seu consentimento a qualquer momento, ou deixar de participar do estudo, sem necessidade de expor suas razões.

Toda informação obtida decorrente dessa pesquisa será submetida aos regulamentos da FOB/USP, referentes ao sigilo da informação. O seu nome será preservado nos resultados ou informações que forem utilizados para fins de publicação científica.

Finalmente, este documento será assinado e rubricado em todas as suas páginas, sendo assinadas 2 vias, permanecendo uma com o(a) senhor(a) paciente e uma com o profissional que está realizando a pesquisa.

Para esclarecimentos de possíveis dúvidas sobre os procedimentos e assuntos relacionados ao estudo, o(a) senhor(a) poderá entrar em contato com o Departamento de Fonoaudiologia da Faculdade de Odontologia de Bauru -FOB/USP, por meio do endereço Alameda Dr. Octávio Pinheiro Brisolla, 9-75, Vila Universitária, telefones (14) 3235-8232 / 3235-8332 ou e-mail: dep-fono@fob.usp.br, ou com apesquisadora Rhaellen Cristine Sevilha Senis e-mail rhaellen.fono@gmail.com. Caso queira apresentar reclamações/denúncias em relação a sua participação no estudo, poderá entrar em contato com o Comitê de Ética em Pesquisa em Seres Humanos da FOB/USP, pelo endereço Al. Dr. Octávio Pinheiro Brisolla, 9-75 (Setor de PósGraduação, piso superior), pelo telefone (14) 3235-8356 ou pelo e-mail: cep@fob.usp.br.

Pelo presente instrumento que atende às exigências legais, o Sr. (a)

, portador da cédula de identidade , após

leitura minuciosa das informações constantes neste TERMO DE CONSENTIMENTO LIVRE E ESCLARECIDO, devidamente explicada pelos profissionais em seus mínimos detalhes, ciente dos serviços e procedimentos aos quais será submetido, não restando quaisquer dúvidas a respeito do lido e explicado, DECLARA e FIRMA seu CONSENTIMENTO LIVRE E ESCLARECIDO concordando em participar da pesquisa proposta. Fica claro que o participante da pesquisa pode a qualquer momento retirar seu CONSENTIMENTO LIVRE E ESCLARECIDO e deixar de participar desta pesquisa e ciente de que todas as informações prestadas tornar-se-ão confidenciais e guardadas por força de sigilo profissional (Art. $13^{\circ}$ do Código de Ética Fonoaudiológico). 
Por fim, como pesquisador(a) responsável pela pesquisa, DECLARO o cumprimento do disposto na Resolução CNS no 466 de 2012, contidos nos itens IV.3, item IV.5.a e na íntegra com a resolução CNS nº 466 de dezembro de 2012.

Por estarmos de acordo com o presente termo o firmamos em duas vias igualmente válidas (uma via para o participante da pesquisa e outra para 0 pesquisador) que serão rubricadas em todas as suas páginas e assinadas ao seu término, conforme o disposto pela Resolução CNS no 466 de 2012, itens IV.3.f e IV.5.d.

Bauru, SP, de de 